\title{
SOME ENERGY CONSERVATIVE SCHEMES FOR VIBRO-IMPACTS OF A BEAM ON RIGID OBSTACLES*
}

\author{
C. Pozzolini ${ }^{1,2}$ and M. Salaun ${ }^{3}$
}

\begin{abstract}
Caused by the problem of unilateral contact during vibrations of satellite solar arrays, the aim of this paper is to better understand such a phenomenon. Therefore, it is studied here a simplified model composed by a beam moving between rigid obstacles. Our purpose is to describe and compare some families of fully discretized approximations and their properties, in the case of non-penetration Signorini's conditions. For this, starting from the works of Dumont and Paoli, we adapt to our beam model the singular dynamic method introduced by Renard. A particular emphasis is given in the use of a restitution coefficient in the impact law. Finally, various numerical results are presented and energy conservation capabilities of the schemes are investigated.
\end{abstract}

Mathematics Subject Classification. 35L85, 65M12, 74H15, 74H45.

Received October 8, 2010.

Published online July 4, 2011.

\section{INTRODUCTION}

During the launch of a satellite, the level of excitation is very high and the mass reduction of satellite solar arrays can lead to impact problem and possibly damage the structure. So CNES is very interested in the use of Finite Element Method (FEM) including local unilateral contact. The goal is to ensure that numerical simulations are predictive enough to maintain a high reliability of spacecraft structures. This problem has been a subject of intense research over the past twenty years, but the introduced methods are still hard to apply on industrial structures. By the way, in industrial FEM analysis, it is usual to make simplifying assumptions, in the modelling of joints for example, or to neglect some phenomena, such as contact between structures. Then, an updating is introduced to modify some parameters (such as mass, stiffness and damping of sub-structures or connections between components) in the numerical model in order to obtain better agreement between numerical and experimental data. To select erroneous parameters, a localization criterion is applied and, classically at

\footnotetext{
Keywords and phrases. Variational inequalities, finite element method, elastic beam, dynamics, unilateral constraints, restitution coefficient.

* The authors would like to thank Pr. Yves Renard for stimulating discussions. This work has been carried out with the generous support of Centre National d'Etudes Spatiales (Toulouse, France).

1 Pôle de Mathématiques, INSA de Lyon, 20 rue Albert Einstein, 69621 Villeurbanne Cedex, France.

cedric.pozzolini@insa-lyon.fr

2 Centre National d'Études Spatiales, 18 avenue Édouard Belin, 31401 Toulouse, France.

3 Université de Toulouse; INSA, UPS, EMAC, ISAE; ICA (Institut Clément Ader); 10 avenue Édouard Belin, 31055 Toulouse,

France. michel.salaun@isae.fr
} 
CNES, a constitutive relation error is used. It means that the mechanical system energy is used. In this respect, it is of importance that the numerical simulations does not affect the energy of the system.

As far as elastodynamic contact problems are concerned, it is known that most of usual numerical schemes exhibit spurious oscillations on the contact displacement and stress (see for instance [7]). Moreover, these oscillations do not disappear when the time step decreases. On the contrary, they tend to increase, which makes very difficult to build stable numerical schemes to solve such problems. These difficulties have already led to many researches and a great variety of solutions were proposed. A first idea is to add damping terms but it leads to a loss of accuracy on the solution. However, let us remark that adding damping leads to some existence results, such as [8]. Another way is to implicit the contact stress (see [2,3]) but the kinetic energy of the contacting nodes is lost at each impact. Looking for some energy conserving schemes is now a well-adressed problem, see for example $[6,9,10,17]$. Nevertheless, these schemes exhibit large oscillations on the contact stress. Besides, most of them do not strictly respect the constraint. Moreover, the way to establish balance of energy is a mathematicaly very difficult problem even in the "simple" case of viscoelastic barmodel with Signorini condition, see $[14,15]$.

So, the goal of this paper is to introduce energy conserving schemes, based on the singular dynamic method introduced by Renard [16], inspired from [7]. As this method was built for second order problems (Laplace operator or elasticity), we try here to achieve a generalization to fourth order problems such as Euler-Bernouilli beams or Kirchhoff-Love plates. Let us remark that this paper will only adress the case of beams but the case of plates is in progress.

Consequently, this paper will be organized as follows. In the next section, the model problem we adress is described. Then, the so-called singular dynamic method is introduced, for which stable singular mass matrices schemes are derived in the case of our beam model. In Section 3, two full discretized schemes (Midpoint and $\beta$-Newmark) are given, with particular emphasis on the way to take into account the restitution coefficient. Finally, Section 4 presents various numerical results and investigates energy conservation capabilities of the previous schemes.

\section{The COntinuous elastodynamic CONTACT PROBlem}

The motion of a beam submitted to an external load is studied. This beam is clamped on its left edge and its vertical displacement is limited by rigid obstacles. Its longitudinal axis, which is its stress free reference configuration, coincides with interval $\Omega=] 0, L[$. Euler-Bernouilli model is chosen to represent the motion of the beam under the assumption of small displacements (the relationship between stress and strain is considered to be linear). Then, $u(x, t)$ stands for the vertical displacement of point $x$ of the beam, at time $t$. In the following, we will use the notations: $u^{\prime}=\frac{\partial u}{\partial x}$ and $\dot{u}=\frac{\partial u}{\partial t}$. So the Euler-Bernouilli model for a clamped/free beam reads

$$
\begin{cases}\rho S \frac{\partial^{2} u}{\partial t^{2}}(x, t)+E I \frac{\partial^{4} u}{\partial x^{4}}(x, t)=f(x, t) & \forall(x, t) \in] 0, L[\times] 0, T[ \\ u(x, 0)=u_{0}(x), \quad \dot{u}(x, 0)=v_{0}(x) & \forall x \in[0, L] \\ u(0, t)=\frac{\partial u}{\partial x}(0, t)=0=\frac{\partial^{3} u}{\partial x^{3}}(L, t)=\frac{\partial^{2} u}{\partial x^{2}}(L, t) & \forall t \in[0, T]\end{cases}
$$

where $\rho\left(\geq \rho_{0}>0\right)$ is the mass density and $E$ is the Young's modulus of the material, while $S$ and $I$ are respectively the surface and the inertial momentum of the beam section. In the case of a sine-sweep base forced vibration, boundary conditions should be given on the left edge by

$$
u(0, t)=a \sin (\omega t), \quad \frac{\partial u}{\partial x}(0, t)=0, \quad \forall t \in[0, T] .
$$


As we will look for a variational solution of (1.1), we need to introduce some functional spaces. So, we set

$$
\mathbb{H}=L^{2}(\Omega), \quad \mathbb{W}=\left\{w \in H^{2}(\Omega) / w(0)=w^{\prime}(0)=0\right\}
$$

where $H^{2}(\Omega)$ is the usual Sobolev space, while the equalities $w(0)=w^{\prime}(0)=0$ should be understood in the trace sense. Moreover, in the case of dynamic frictionless Euler-Bernouilli model with Signorini contact conditions along the beam, the displacement has to belong to the convex set $\mathbb{K} \subset \mathbb{W}$ given by

$$
\mathbb{K}=\left\{w \in \mathbb{W} / g_{1}(x) \leq w(x) \leq g_{2}(x), \quad \forall x \in[0, L]\right\},
$$

where $g_{1}$ and $g_{2}$ are two mappings from $[0, L]$ to $\overline{\mathbb{R}}$ such that there exists $g>0$ such that

$$
g_{1}(x) \leq-g<0<g \leq g_{2}(x) \quad \forall x \in[0, L] .
$$

Then, the mechanical frictionless elastodynamic problem for a beam between two rigid obstacles can be written as the following variational inequality

$$
\left\{\begin{array}{l}
\text { Find } u:[0, T] \rightarrow \mathbb{K} \text { such that for almost every } t \in[0, T] \text { and for every } w \in \mathbb{W} \\
\int_{\Omega}\left[\rho S \frac{\partial^{2} u}{\partial t^{2}}(t)(w-u(t))+E I \frac{\partial^{2} u}{\partial x^{2}}(t) \frac{\partial^{2}(w-u(t))}{\partial x^{2}}\right] \mathrm{d} \Omega \geq \int_{\Omega} f(w-u(t)) \mathrm{d} \Omega \\
u(x, 0)=u_{0}(x) \in \mathbb{K}, \quad \dot{u}(x, 0)=v_{0}(x), \quad \forall x \in \Omega .
\end{array}\right.
$$

Assuming that $f \in L^{2}(0, T ; \mathbb{H}), u_{0} \in \mathbb{K}, v_{0} \in \mathbb{H}$, Kuttler and Schillor proved in [8] that problem (1.3) has a solution $u$ belonging to $L^{2}(0, T ; \mathbb{K})$. For this, they used a penalty method. Another proof of this result is due to Dumont and Paoli [4], who established convergence of the solutions of fully discretized approximations of the problem. As far as uniqueness is concerned, it can be easily shown that it does not occur for (1.3): a counterexample is given in [1]. Finally, there is no result about conservation of energy at the limit.

In fact, discretization of (1.3) does not describe completely the motion: a constitutive law for impact should be added (see [11]). For example, if there is an impact at $\left(x_{0}, t_{0}\right)$, this law gives the relation between velocities before and after impact

$$
\dot{u}\left(x_{0}, t_{0}^{+}\right)=-e \dot{u}\left(x_{0}, t_{0}^{-}\right) \quad \text { whenever } u\left(x_{0}, t_{0}\right) \in \partial \mathbb{K},
$$

where scalar $e$, called restitution coefficient, is a real number belonging to $[0,1] . e=1$ matches to a perfect impact: velocity is conserved, up to its sign, whereas $e=0$ is an absorbing shock. Let us remark that, in [13], the authors show that the restitution coefficient for a bar is a rather ill-defined concept. For instance, their numerical experiments underline the observed restitution coefficient depends very strongly on the initial angle of the bar with horizontal. Moreover, in the particular case of a slender bar dropped on a rigid foundation, the chosen value of the restitution coefficient does not seem to have a great influence on the displacement limit when the space step tends to zero.

Despite the previous remarks, our idea is to explicitly incorporate the restitution coefficient into (1.3) and to observe how some numerical schemes will simulate the experimental behavior of a vibrating beam. Doing this, our aim is to investigate the possibility to use restitution coefficient as an unknown parameter for updating of FEM with experimental results.

\section{Singular Dynamic Method}

\subsection{Well-posed space semi-discretization}

The goal of this section is to present a well-posed space semi-discretization of Problem (1.3). As usual, a Galerkin method is used for space discretization, but the original idea, due to Renard [16], is to introduce 
different approximations for displacement $u$ and velocity $v=\dot{u}$. So, let $\mathbb{W}^{h}$ and $\mathbb{H}^{h}$ be two finite dimensional vector subspaces of $\mathbb{W}$ and $\mathbb{H}$ respectively. Let $\mathbb{K}^{h} \subset \mathbb{W}^{h}$ be a closed convex nonempty approximation of $\mathbb{K}$. The new approximation of (1.3) reads

$$
\left\{\begin{array}{l}
\text { Find } u^{h}:[0, T] \rightarrow \mathbb{K}^{h} \text { and } v^{h}:[0, T] \rightarrow \mathbb{H}^{h} \text { such that for all } t \in(0, T] \\
\int_{\Omega}\left[\rho S \frac{\partial v^{h}}{\partial t}\left(w^{h}-u^{h}\right)+E I \frac{\partial^{2} u^{h}}{\partial x^{2}} \frac{\partial^{2}\left(w^{h}-u^{h}\right)}{\partial x^{2}}\right] \mathrm{d} \Omega \geq \int_{\Omega} f\left(w^{h}-u^{h}\right) d \Omega, \forall w^{h} \in \mathbb{K}^{h} \\
\int_{\Omega} \rho S\left[v^{h}-\frac{\partial u^{h}}{\partial t}\right] q^{h} \mathrm{~d} \Omega=0, \quad \forall q^{h} \in \mathbb{H}^{h} \\
u^{h}(x, 0)=u_{0}^{h}(x), \quad v^{h}(x, 0)=v_{0}^{h}(x), \quad \forall x \in \Omega
\end{array}\right.
$$

where $u_{0}^{h} \in \mathbb{K}^{h}$ and $v_{0}^{h} \in \mathbb{H}^{h}$ are approximations of $u_{0}$ and $v_{0}$ respectively. The case $\mathbb{H}^{h}=\mathbb{W}^{h}$ clearly corresponds to a standard Galerkin approximation of (1.3).

Let us now introduce some basis of $\mathbb{W}^{h}$ and $\mathbb{H}^{h}$, say respectively $\phi_{i}\left(1 \leq i \leq N_{W}\right)$ and $\psi_{i}\left(1 \leq i \leq N_{H}\right)$. The above discrete variational formulation is associated with matrices $\mathbf{K}$ (stiffness matrix), $\mathbf{B}$ and $\mathbf{C}$ (mass matrices) of respective sizes $N_{W}^{2}, N_{H} \times N_{W}$ and $N_{H}^{2}$, defined by

$$
\mathbf{K}_{i j}=\int_{\Omega} E I \phi_{i}^{\prime \prime} \phi_{j}^{\prime \prime} \mathrm{d} \Omega, \quad \mathbf{B}_{i j}=\int_{\Omega} \rho S \phi_{i} \psi_{j} \mathrm{~d} \Omega, \quad \mathbf{C}_{i j}=\int_{\Omega} \rho S \psi_{i} \psi_{j} \mathrm{~d} \Omega .
$$

The related vectors, say $F, U$ (components $u_{i}$ ) and $V$ (components $v_{i}$ ), of size $N_{W}, N_{W}$ and $N_{H}$ respectively, are such that

$$
F_{i}=\int_{\Omega} f \phi_{i} \mathrm{~d} \Omega, \quad u^{h}(t)=\sum_{i=1}^{N_{W}} u_{i}(t) \phi_{i}, \quad v^{h}(t)=\sum_{i=1}^{N_{H}} v_{i}(t) \psi_{i} .
$$

Let us remark that the second equation of (2.1) reads

$$
\mathbf{C} V(t)=\mathbf{B} \dot{U}(t)
$$

Since $\mathbf{C}$ is always invertible, we obtain $V(t)=\mathbf{C}^{-1} \mathbf{B} \dot{U}(t)$ and, then, $\dot{V}(t)=\mathbf{C}^{-1} \mathbf{B} \ddot{U}(t)$, which allows to eliminate $V$. So the semi-discretized problem (2.1) is equivalent to

$$
\left\{\begin{array}{l}
\text { Find } U:[0, T] \rightarrow \mathbb{K}^{h} \text { and } V:[0, T] \rightarrow \mathbb{H}^{h} \text { such that for all } t \in(0, T] \\
(W-U(t))^{T}(\mathbf{M} \ddot{U}(t)+\mathbf{K} U(t)) \geq(W-U(t))^{T} F, \quad \forall W \in \mathbb{K}^{h}, \\
\mathbf{C} V(t)=\mathbf{B} \dot{U}(t), \\
U(0)=U_{0}, \quad V(0)=V_{0},
\end{array}\right.
$$

where $\mathbf{M}$ is the so-called singular mass matrix defined by

$$
\mathbf{M}=\mathbf{B}^{T} \mathbf{C}^{-1} \mathbf{B}
$$

Let us now explain how the approximation $\mathbb{K}^{h}$ of $\mathbb{K}$ is obtained. We recall that

$$
\mathbb{K}=\left\{w \in \mathbb{W} / g_{1}(x) \leq w(x) \leq g_{2}(x), \quad \forall x \in[0, L]\right\}
$$


As usual, it is natural to introduce a partition of interval $[0, L]$ into $N$ subintervals of length $h=L / N$, built on nodes $x_{i}=i h$, for $0 \leq i \leq N$. So, unilateral constraints are only considered at these nodes. It means convex $\mathbb{K}^{h}$ is

$$
\mathbb{K}^{h}=\left\{w^{h} \in \mathbb{W}^{h} / g_{1}\left(x_{i}\right) \leq w^{h}\left(x_{i}\right) \leq g_{2}\left(x_{i}\right), \quad \forall i \in[0, N]\right\} .
$$

With vector notations, setting $\alpha_{i}^{-} \equiv g_{1}\left(x_{i}\right)$ and $\alpha_{i}^{+} \equiv g_{2}\left(x_{i}\right)$ for all $i$, this space may be written (we keep the same notation for simplicity)

$$
\mathbb{K}^{h}=\left\{W \in \mathbb{R}^{N_{W}} / \alpha_{i}^{-} \leq\left(G^{i}\right)^{T} W \leq \alpha_{i}^{+}, \quad \forall i \in[0, N]\right\},
$$

where $G^{i}$ is the vector of $\mathbb{R}^{N_{W}}$ such that $\left(G^{i}\right)^{T} W=w^{h}\left(x_{i}\right)$, for all node $x_{i}$.

Remark 2.1. Since we deal with a fourth order problem with respect to the space derivative, it is not possible to consider a linear space approximation. In fact, for this beam model, we use the classical Hermite third degree polynomials to approximate the numerical displacement. It means the degrees of freedom are node displacements and their derivatives. So, in the above approximation of $\mathbb{K}$, we consider only constraints on node displacements: the effect of the derivatives, namely the curvature, is not taken into account. Then, in this framework, the beam could cross the obstacle between two nodes, but we shall neglect this aspect in the following.

Furthemore, it was told that functions $g_{1}$ and $g_{2}$ takes their values in $\overline{\mathbb{R}}$, which means that $\alpha_{i}^{ \pm}$may be equal to $\pm \infty$. In this case, the constraint is worthless. For instance, it will be the case if the obstacles are reduced to end stops on the free edge of the beam. Then, $\alpha_{i}^{ \pm}= \pm \infty$ for all $i \neq N$ and $\alpha_{N}^{ \pm}= \pm g$, if $g$ stands for the allowed maximal displacement. Moreover, it is assumed that the clamped edge, which corresponds to node $x_{0}$, satisfies the constraints. So, it is natural to introduce the number, say $N_{G}$, of "real" constraints. For example, $N_{G}=1$ for end stops and $N_{G}=N$ for flat obstacles up and under the beam.

Now, let us denote by $\mathbf{G}$ the $N_{W} \times N_{G}$ matrix, which components are $\mathbf{G}_{i j}=\left(G^{i}\right)_{j}$. As the previous choice is clearly such that vectors $G^{i}$ are linearly independent, using the Lagrange multipliers, the discrete problem (2.2) is also equivalent to the following one

$$
\left\{\begin{array}{l}
\text { Find } U:[0, T] \rightarrow \mathbb{K}^{h} \text { and } V:[0, T] \rightarrow \mathbb{H}^{h} \text { such that for all } t \in(0, T] \\
\mathbf{M} \ddot{U}(t)+\mathbf{K} U(t)=F+\sum_{i=1}^{N_{G}} \lambda^{i}(t) G^{i}, \\
\left\{\begin{array}{l}
\lambda^{i}(t) \geq 0, \quad\left(G^{i}\right)^{T} U(t) \geq \alpha_{i}^{-}, \quad \lambda^{i}(t)\left(\left(G^{i}\right)^{T} U(t)-\alpha_{i}^{-}\right)=0 \\
\text { or } \\
\lambda^{i}(t) \leq 0, \quad\left(G^{i}\right)^{T} U(t) \leq \alpha_{i}^{+}, \quad \lambda^{i}(t)\left(\left(G^{i}\right)^{T} U(t)-\alpha_{i}^{+}\right)=0
\end{array}\right\} 1 \leq i \leq N_{G} \\
\mathbf{C} V(t)=\mathbf{B} \dot{U}(t), \\
U(0)=U_{0}, \quad V(0)=V_{0} .
\end{array}\right.
$$

Here, the Lagrange multipliers $\lambda^{i}$ are the reaction forces which are measures on $(0, T]$. And the orthogonality has its natural meaning: an appropriate duality product between the two terms of the relation vanishes.

Now, let us introduce subspace $\mathbb{F}^{h}$ of $\mathbb{W}^{h}$, defined by

$$
\mathbb{F}^{h}=\left\{w^{h} \in \mathbb{W}^{h} / \int_{\Omega} \rho S w^{h} \xi^{h} d \Omega=0, \quad \forall \xi^{h} \in \mathbb{H}^{h}\right\} .
$$

Then, with the above definitions, we have

$$
\mathbb{F}^{h}=\operatorname{ker} \mathbf{B} .
$$

The proof of the following result can be found in [16]. 
Theorem 2.2. If $\mathbb{W}^{h}, \mathbb{H}^{h}$ and $\mathbb{K}^{h}$ satisfy the following Inf-Sup condition

$$
\inf _{Q \in \mathbb{R}^{N_{g}} \backslash\{0\}} \sup _{\mathbb{W} \in \mathbb{F}^{h} \backslash\{0\}} \frac{Q^{T} \mathbf{G} W}{\|Q\|\|W\|}>0,
$$

then Problem (2.2) admits a unique solution $U(t)$. Moreover, this solution is Lipschitz-continuous with respect to $t$ and verifies the following persistency condition

$$
\lambda^{i}(t)\left(G^{i}\right)^{T} \dot{U}(t)=0, \quad \forall t \in(0, T], \quad 1 \leq i \leq N_{g} .
$$

Finally, solution $U(t)$ is energy conserving in the sense that the discrete energy

$$
E^{h}(t)=\frac{1}{2} \dot{U}^{T}(t) \mathbf{M} \dot{U}(t)+\frac{1}{2} U^{T}(t) \mathbf{K} U(t)-U^{T}(t) F,
$$

is constant with respect to $t$.

Remark 2.3. The persistency condition (see [9] and [10]), which links velocity $\dot{U}(t)$ and Lagrange multipliers, is a stronger condition than the classical complementary condition between solution $U(t)$ and Lagrange multipliers. That is this persistency condition which allows to prove energy conservation.

\subsection{Numerical discretization}

Thanks to the above theorem, proving condition (2.6) is sufficient to obtain well-posedness of the discrete problem (2.2). Let us remark this condition is equivalent to the fact that matrix $\mathbf{G}$ is surjective on $\mathbb{F}^{h}$. As a result of this, we must have

$$
\operatorname{dim} \mathbb{F}^{h} \geq N_{G} \quad \text { and consequently } \quad \operatorname{dim} \mathbb{H}^{h} \leq \operatorname{dim} \mathbb{W}^{h}-N_{G}
$$

This prescribes conditions on the approximation spaces $\mathbb{W}^{h}, \mathbb{H}^{h}$ and also $\mathbb{K}^{h}$. In order to illustrate that condition (2.6) holds for interesting practical situations, we will give two examples of approximation spaces for our beam problem.

To build the finite element method, it was introduced a partition of $[0, L]$ into $N$ subintervals of length $h=L / N$, built on nodes $x_{i}=i h$, for $0 \leq i \leq N$. As node $x_{0}=0$ is clamped, we will omit it from now on and consider that index $i$ varies between 1 and $N$. Otherwise, it would introduce small modifications in the following. So, at each node $x_{i}$ are associated two Hermite piecewise cubic functions, say $\phi_{2 i-1}$ and $\phi_{2 i}$, defined for $1 \leq i \leq N$ by

$$
\phi_{2 i-1}\left(x_{j}\right)=\delta_{i j} \quad \text { and } \quad \phi_{2 i-1}^{\prime}\left(x_{j}\right)=0, \quad \phi_{2 i}\left(x_{j}\right)=0 \quad \text { and } \quad \phi_{2 i}^{\prime}\left(x_{j}\right)=\delta_{i j},
$$

where $\delta_{i j}$ is Kronecker symbol. Moreover, functions $\phi_{j}$ are chosen of class $\mathcal{C}^{1}$ on $[0, L]$, which insures that each $\phi_{j}$ belongs to the continuous space $\mathbb{W}$. Hence, displacement $w^{h}$ reads

$$
w^{h}(x)=\sum_{i=1}^{N} w_{2 i-1}^{h} \phi_{2 i-1}(x)+\sum_{i=1}^{N} w_{2 i}^{h} \phi_{2 i}(x),
$$

and coefficient $w_{2 i-1}^{h}$ gives the value of $w^{h}$ at node $x_{i}$ while $w_{2 i}^{h}$ gives the value of its derivative at the same node. The approximation space for displacements is then

$$
\mathbb{W}^{h}=\operatorname{span}\left\{\phi_{j}, 1 \leq j \leq 2 N\right\}
$$

which is a subset of $\mathbb{W}$. In this case, with the previous notations, we have $N_{W}=2 N$. 
Now, let us recall that constraints are only considered at nodes $x_{i}$. If $w^{h}$ is an element of $\mathbb{W}^{h}$, we have

$$
\mathbb{K}^{h}=\left\{w^{h} \in \mathbb{W}^{h} / \alpha_{i}^{-} \leq w^{h}\left(x_{i}\right)=w_{2 i-1}^{h} \leq \alpha_{i}^{+}, \quad \forall i \in[1, N]\right\} .
$$

Let $W$ be the vector of $\mathbb{R}^{N_{W}}$ which components are $w_{j}^{h}$. Then, vector $G^{i}$ of $\mathbb{R}^{N_{W}}$, which components are all zero except $\left(G^{i}\right)_{2 i-1}=1$, is such that $\left(G^{i}\right)^{T} W=w_{2 i-1}^{h}$, for all node $x_{i}$. To check the Inf-Sup condition, we will consider the "worst" case, which occurs when the whole beam is between two obstacles. It means that each $\alpha_{i}^{ \pm}$is finite.

\subsection{1. $P_{0}$ interpolation for velocity}

The first choice for space $\mathbb{H}^{h}$ is to use piecewise constant polynomial functions. Let us begin by characterizing $\mathbb{F}^{h}=\operatorname{ker} \mathbf{B}$. Let $w^{h}$ be an element of $\mathbb{W}^{h}$. We have

$$
w^{h}(x)=\sum_{i=1}^{N} w_{2 i-1}^{h} \phi_{2 i-1}(x)+\sum_{i=1}^{N} w_{2 i}^{h} \phi_{2 i}(x) .
$$

$w^{h}$ belongs to $\mathbb{F}^{h}$ if

$$
\int_{\Omega} \rho S w^{h} \xi^{h} \mathrm{~d} \Omega=0, \quad \forall \xi^{h} \in \mathbb{H}^{h} .
$$

In the following, we will assume that $\rho S$ is constant all along the beam, which allows to drop it. Then, the previous relation is equivalent to

$$
\int_{x_{i-1}}^{x_{i}} w^{h} \mathrm{~d} x=0, \quad \forall i \in[1, N] .
$$

For $i=1$, it becomes $\int_{x_{0}}^{x_{1}}\left(w_{1}^{h} \phi_{1}(x)+w_{2}^{h} \phi_{2}(x)\right) \mathrm{d} x=0$. As $x_{0}=0$ and $x_{1}=h$ (mesh size), it is easy to check that $\phi_{2}(x)=\frac{x^{2}}{h^{2}}(x-h)$ and $\int_{0}^{h} \phi_{2}(x) \mathrm{d} x=-\frac{h^{2}}{12}$, which is always non zero. So, it is possible to compute $w_{2}^{h}$ from $w_{1}^{h}$ and we have

Similarly, for $i \neq 1$, we have

$$
w_{2}^{h}=-w_{1}^{h} \frac{\int_{x_{0}}^{x_{1}} \phi_{1}(x) \mathrm{d} x}{\int_{x_{0}}^{x_{1}} \phi_{2}(x) \mathrm{d} x}
$$

$$
\int_{x_{i-1}}^{x_{i}}\left(w_{2 i-3}^{h} \phi_{2 i-3}(x)+w_{2 i-2}^{h} \phi_{2 i-2}(x)+w_{2 i-1}^{h} \phi_{2 i-1}(x)+w_{2 i}^{h} \phi_{2 i}(x)\right) \mathrm{d} x=0,
$$

which leads to

$$
w_{2 i}^{h}=-w_{2 i-3}^{h} \frac{\int_{x_{i-1}}^{x_{i}} \phi_{2 i-3}(x) \mathrm{d} x}{\int_{x_{i-1}}^{x_{i}} \phi_{2 i}(x) \mathrm{d} x}-w_{2 i-2}^{h} \frac{\int_{x_{i-1}}^{x_{i}} \phi_{2 i-2}(x) \mathrm{d} x}{\int_{x_{i-1}}^{x_{i}} \phi_{2 i}(x) \mathrm{d} x}-w_{2 i-1}^{h} \frac{\int_{x_{i-1}}^{x_{i}} \phi_{2 i-1}(x) \mathrm{d} x}{\int_{x_{i-1}}^{x_{i}} \phi_{2 i}(x) \mathrm{d} x},
$$

as $\int_{x_{i-1}}^{x_{i}} \phi_{2 i}(x) \mathrm{d} x \neq 0$ for the same reason than above. Consequently, as $w_{2}^{h}$ is function of $w_{1}^{h}, w_{4}^{h}$ depends on $w_{1}^{h}$ and $w_{3}^{h}$ and, by direct induction, for any element of $\operatorname{ker} \mathbf{B}$, the degrees of freedom $w_{2 i}^{h}$ can be expressed as functions of $w_{2 j-1}^{h}$. It means any element $\bar{w}^{h}(x)$ of ker $\mathbf{B}$ reads

$$
\bar{w}^{h}(x)=\sum_{i=1}^{N} w_{2 i-1}^{h} \phi_{2 i-1}(x)+\sum_{i=1}^{N} f_{i}\left(w_{2 j-1}^{h}\right) \phi_{2 i}(x),
$$

where $f_{i}\left(w_{2 j-1}^{h}\right)$ is a function of the "odd" degrees of freedom. 
Therefore, if $\bar{W}$ is the vector of $\mathbb{R}^{N_{W}}$ which components are $\bar{w}_{j}^{h}$, it becomes obvious that $\left(G^{i}\right)^{T} \bar{W}^{\prime}=w_{2 i-1}^{h}$, for all node $x_{i}$ : $\mathbf{G}$ is surjective on ker $\mathbf{B}$ and the Inf-Sup condition is satisfied.

Remark 2.4. It is easy to see the previous proof remains true if the mesh size $h$ is not constant, which means nodes $x_{i}$ are not regularly distributed along the beam. Similarly, the condition " $\rho S$ constant all along the beam" may be replaced by " $\rho S$ constant on each element".

\subsection{2. $P_{1}$ interpolation for the velocity}

The second choice for space $\mathbb{H}^{h}$ is to use continuous piecewise linear polynomial functions. If $N+1$ is the number of nodes on the beam (including the "clamped node"), let us remark that we have in this case $\operatorname{dim} \mathbb{H}^{h}=N+1$ which is not less equal than $\operatorname{dim} \mathbb{W}^{h}-N_{G}=2 N-N=N$ in the worst case. Nevertheless, the "clamped node" velocity is zero and it is natural to take this condition into account. Then, $\operatorname{dim} \mathbb{H}^{h}=N$ and we can hope the Inf-Sup condition may occur.

As above, let us begin by characterizing $\mathbb{F}^{h}$. Starting from definitions of $w^{h}(2.7)$ and $\mathbb{F}^{h}(2.8)$, and assuming again that $\rho S$ is constant which allows to drop it, we obtain

$$
\int_{x_{i-1}}^{x_{i+1}} w^{h} \psi_{i} \mathrm{~d} x=0, \quad \forall i \in[1, N-1],
$$

and

$$
\int_{x_{N-1}}^{x_{N}} w^{h} \psi_{N} \mathrm{~d} x=0,
$$

where $\psi_{i}$ is the continuous piecewise linear function such that $\psi_{i}\left(x_{j}\right)=\delta_{i j}$. Before analysing these equations, let us first give the expressions of the finite element basis functions, we shall need further. It is made on interval $[0, h]$, the extension to a generic interval $\left[x_{i}, x_{i+1}\right]$, where $x_{i}=i h$, being obtained by a simple translation. So we have for the piecewise linear polynomial functions

$$
\psi_{l}(x)=1-\frac{x}{h}, \quad \psi_{r}(x)=\frac{x}{h}
$$

and for the third order polynomial functions associated with the derivative degrees of freedom

$$
\phi_{l}(x)=x\left(1-\frac{x}{h}\right)^{2}, \quad \phi_{r}(x)=\frac{x^{2}}{h^{2}}(x-h)
$$

where indices $l$ and $r$ represent the left node and the right one respectively. Finally, it is easy to check that

$$
\left\{\begin{array}{l}
\int_{0}^{h} \phi_{l}(x) \psi_{l}(x) \mathrm{d} x=\frac{h^{2}}{20}=-\int_{0}^{h} \phi_{r}(x) \psi_{r}(x) \mathrm{d} x \\
\int_{0}^{h} \phi_{l}(x) \psi_{r}(x) \mathrm{d} x=\frac{h^{2}}{30}=-\int_{0}^{h} \phi_{r}(x) \psi_{l}(x) \mathrm{d} x .
\end{array}\right.
$$

Then, for $i=1$, by using (2.11), equation (2.9) becomes

$$
\begin{aligned}
\int_{x_{0}}^{x_{2}} w^{h} \psi_{1} \mathrm{~d} x & =\int_{x_{0}}^{x_{2}}\left(w_{1}^{h} \phi_{1}+w_{2}^{h} \phi_{2}\right) \psi_{1} \mathrm{~d} x+\int_{x_{1}}^{x_{2}}\left(w_{3}^{h} \phi_{3}+w_{4}^{h} \phi_{4}\right) \psi_{1} \mathrm{~d} x \\
& =w_{1}^{h} \int_{x_{0}}^{x_{2}} \phi_{1} \psi_{1} \mathrm{~d} x+w_{2}^{h}\left(-\frac{h^{2}}{20}+\frac{h^{2}}{20}\right)+w_{3}^{h} \int_{x_{1}}^{x_{2}} \phi_{3} \psi_{1} \mathrm{~d} x-w_{4}^{h} \frac{h^{2}}{30} \\
& =0 .
\end{aligned}
$$


So, it is possible to compute $w_{4}^{h}$ from $w_{1}^{h}$ and $w_{3}^{h}$ as $w_{4}^{h}=f\left(w_{1}^{h}, w_{3}^{h}\right)$. For simplicity, in the following, $f$ will indicate various (polynomial) functions. Similarly, for $1<i<N$, we have

$$
\begin{aligned}
\int_{x_{i-1}}^{x_{i+1}} w^{h} \psi_{i} \mathrm{~d} x & =\int_{x_{i-1}}^{x_{i}}\left(w_{2 i-3}^{h} \phi_{2 i-3}+w_{2 i-2}^{h} \phi_{2 i-2}\right) \psi_{i} \mathrm{~d} x+\int_{x_{i-1}}^{x_{i+1}}\left(w_{2 i-1}^{h} \phi_{2 i-1}+w_{2 i}^{h} \phi_{2 i}\right) \psi_{i} \mathrm{~d} x \\
& +\int_{x_{i}}^{x_{i+1}}\left(w_{2 i+1}^{h} \phi_{2 i+1}+w_{2 i+2}^{h} \phi_{2 i+2}\right) \psi_{i} \mathrm{~d} x \\
& =w_{2 i-3}^{h} \int_{x_{i-1}}^{x_{i}} \phi_{2 i-3} \psi_{i} \mathrm{~d} x+w_{2 i-2}^{h} \frac{h^{2}}{30}+w_{2 i-1}^{h} \int_{x_{i-1}}^{x_{i+1}} \phi_{2 i-1} \psi_{i} \mathrm{~d} x \\
& +w_{2 i}^{h}\left(-\frac{h^{2}}{20}+\frac{h^{2}}{20}\right)+w_{2 i+1}^{h} \int_{x_{i}}^{x_{i+1}} \phi_{2 i+1} \psi_{i} \mathrm{~d} x-w_{2 i+2}^{h} \frac{h^{2}}{30} \\
& =0
\end{aligned}
$$

which leads to

$$
w_{2 i+2}^{h}=w_{2 i-2}^{h}+f\left(w_{2 i-3}^{h}, w_{2 i-1}^{h}, w_{2 i+1}^{h}\right) .
$$

Consequently, by direct induction, we obtain

$$
\left\{\begin{aligned}
w_{2}^{h} & =w_{2}^{h} \\
w_{4}^{h} & =f\left(w_{1}^{h}, w_{3}^{h}\right) \\
w_{6}^{h} & =w_{2}^{h}+f\left(w_{1}^{h}, w_{3}^{h}, w_{5}^{h}\right) \\
w_{8}^{h} & =w_{4}^{h}+f\left(w_{3}^{h}, w_{5}^{h}, w_{7}^{h}\right)=f\left(w_{1}^{h}, w_{3}^{h}, w_{5}^{h}, w_{7}^{h}\right) \\
w_{10}^{h} & =w_{6}^{h}+f\left(w_{5}^{h}, w_{7}^{h}, w_{9}^{h}\right)=w_{2}^{h}+f\left(w_{1}^{h}, w_{3}^{h}, w_{5}^{h}, w_{7}^{h}, w_{9}^{h}\right) \\
\cdots & \\
w_{4 k-2}^{h} & =w_{2}^{h}+f\left(w_{\text {odd }}^{h}\right) \\
w_{4 k}^{h} & =f\left(w_{\text {odd }}^{h}\right) \\
\cdots &
\end{aligned}\right.
$$

where $f\left(w_{\text {odd }}^{h}\right)$ stands for various functions depending on the set of $w_{j}^{h}, j$ being odd. In particular, let us observe that the two last equations, corresponding to $i=N-1$, read

$$
\left\{\begin{aligned}
\left\{\begin{aligned}
w_{2 N-2}^{h}=w_{2}^{h}+f\left(w_{o d d}^{h}\right) \\
w_{2 N}^{h}=f\left(w_{o d d}^{h}\right)
\end{aligned}\right\} & \text { if } N \text { is even } \\
\left\{\begin{aligned}
w_{2 N-2}^{h}=f\left(w_{o d d}^{h}\right) \\
w_{2 N}^{h}=w_{2}^{h}+f\left(w_{\text {odd }}^{h}\right)
\end{aligned}\right\} & \text { if } N \text { is odd. }
\end{aligned}\right.
$$

Finally, the last equation (2.10) becomes

$$
\begin{aligned}
\int_{x_{N-1}}^{x_{N}} w^{h} \psi_{N} \mathrm{~d} x & =\int_{x_{N-1}}^{x_{N}}\left(w_{2 N-3}^{h} \phi_{2 N-3}+w_{2 N-2}^{h} \phi_{2 N-2}+w_{2 N-1}^{h} \phi_{2 N-1}+w_{2 N}^{h} \phi_{2 N}\right) \psi_{N} \mathrm{~d} x \\
& =w_{2 N-3}^{h} \int_{x_{N-1}}^{x_{N}} \phi_{2 N-3} \psi_{N} \mathrm{~d} x+w_{2 N-2}^{h} \frac{h^{2}}{30}+w_{2 N-1}^{h} \int_{x_{N-1}}^{x_{N+1}} \phi_{2 N-1} \psi_{N} \mathrm{~d} x \\
& -w_{2 N}^{h} \frac{h^{2}}{20} \\
& =0,
\end{aligned}
$$

or else

$$
w_{2 N}^{h}=\frac{2}{3} w_{2 N-2}^{h}+f\left(w_{2 N-3}^{h}, w_{2 N-1}^{h}\right) .
$$

Then, using (2.12), in both cases ( $N$ being odd or even), we obtain $w_{2}^{h}=f\left(w_{\text {odd }}^{h}\right)$. 
Finally, exactly as in Section 2.2.1, for any element of ker $\mathbf{B}$, the degrees of freedom $w_{2 i}^{h}$ can be expressed as functions of $w_{2 j-1}^{h}$ and the Inf-Sup condition is satisfied.

\section{Full Discretized schemes}

In this section, we present two approaches for space discretization of the velocity, and we compare Midpoint and $\beta$-Newmark schemes for time discretization. These schemes are interesting since they are energy conserving during the linear part of the motion (equation without constraint). As previously mentioned, classical $P_{3^{-}}$ Hermite finite elements are used to approximate displacement $u$. Moreover, $\Delta t$ will be the time step and $e$ the restitution coefficient.

\subsection{Newmark-Dumont-Paoli schemes}

\subsubsection{Case of a regular mass matrix}

To solve problem (1.3), Dumont-Paoli [4] introduced the following fully implicit Newmark scheme

$$
\left\{\begin{array}{c}
\text { Find } U^{n+1} \in \mathbb{K}^{h} \text { such that for all } W \in \mathbb{K}^{h} \\
\begin{array}{c}
\left(W-U^{n+1}\right)^{T}\left(\mathbf{M}_{r} \frac{U^{n+1}-2 U^{n}+U^{n-1}}{\Delta t^{2}}+\mathbf{K}\left(\beta U^{n+1}+(1-2 \beta) U^{n}+\beta U^{n-1}\right)\right) \\
\geq\left(W-U^{n+1}\right)^{T} F^{n, \beta}
\end{array}
\end{array}\right.
$$

where

$$
F^{n, \beta}=\beta F^{n+1}+(1-2 \beta) F^{n}+\beta F^{n-1},
$$

$F^{k}$ being the vector which components are $F_{i}^{k}=\int_{\Omega} f(x, k \Delta t) \phi_{i}(x) \mathrm{d} \Omega$. Finally, $\left(\phi_{i}\right)_{i}$ stand for the piecewise cubic basis functions defining space $\mathbb{W}^{h}$ and $\mathbf{M}_{r}$ is the associated regular mass matrix.

To take into account the restitution coefficient $e$ defined by (1.4), we follow the choice introduced by Paoli-Schatzman (see $[12,13]$ ), which consists in replacing $U^{n+1}$ by $\frac{U^{n+1}+e U^{n-1}}{1+e}$. Then, a more general discretization of (1.3) becomes

$$
\left\{\begin{array}{c}
\text { Find } U^{n+1, e} \equiv \frac{U^{n+1}+e U^{n-1}}{1+e} \in \mathbb{K}^{h} \text { such that for all } W \in \mathbb{K}^{h} \\
\left(W-U^{n+1, e}\right)^{T}\left(\mathbf{M}_{r} \frac{U^{n+1}-2 U^{n}+U^{n-1}}{\Delta t^{2}}+\mathbf{K}\left(\beta U^{n+1}+(1-2 \beta) U^{n}+\beta U^{n-1}\right)\right) \\
\geq\left(W-U^{n+1, e}\right)^{T} F^{n, \beta}
\end{array}\right.
$$

Let us remark that Dumont-Paoli fully implicit scheme corresponds to $e=0$, which is a totally absorbing impact. In this case, the authors established unconditional stability for $\beta=1 / 2$ whereas a conditional stability result is obtained when $\beta \in[0,1 / 2[$ (see [4]). Moreover, for $\beta \in[0,1 / 2]$, a weak convergence result (up to a subsequence) is demonstrated.

Remark 3.1. Defining the total energy by

$$
E(w, \dot{w}):=\int_{\Omega}\left[\frac{\rho S}{2}(\dot{w}(x, t))^{2}+\frac{E I}{2}\left(w^{\prime \prime}(x, t)\right)^{2}-f(x, t) w(x, t)\right] \mathrm{d} \Omega
$$


it is easy to see that $E\left(u^{n+1}, v^{n+1}\right) \leq E\left(u^{n}, v^{n}\right)$. This Newmark-Dumont-Paoli scheme is dissipative in energy. Indeed, energy is conserved as long as the beam does not touch the obstacles and it is dissipated when beam reaches them, except for a totally elastic shock $(e=1)$ which is the only case where kinetic energy is conserved.

\subsubsection{Case of singular mass matrix}

To derive a Newmark scheme using the singular mass matrix approach, let us go from the equilibrium equation given in $(2.5)$

$$
\mathbf{M} \ddot{U}+\mathbf{K} U=F+\Lambda \equiv \tilde{F},
$$

where $\Lambda$ stands for the reaction, which is zero when there is no contact. Moreover, the singular mass approach introduces matrices $\mathbf{C}$ and $\mathbf{B}$ such that $\mathbf{C} V=\mathbf{B} \dot{U}$, the singular mass matrix being $\mathbf{M}=\mathbf{B}^{T} \mathbf{C}^{-1} \mathbf{B}$.

The usual $(1 / 2, \beta)$-Newmark scheme reads

$$
\left\{\begin{array}{l}
U^{n+1}=U^{n}+\Delta t \dot{U}^{n}+\left(\frac{1}{2}-\beta\right) \Delta t^{2} \ddot{U}^{n}+\beta \Delta t^{2} \ddot{U}^{n+1} \\
\dot{U}^{n+1}=\dot{U}^{n}+\frac{\Delta t}{2} \ddot{U}^{n}+\frac{\Delta t}{2} \ddot{U}^{n+1}
\end{array}\right.
$$

Multiplying left by $\mathbf{B}$ and using $\mathbf{C} V=\mathbf{B} \dot{U}$, we deduce

$$
\left\{\begin{array}{l}
\mathbf{B} U^{n+1}=\mathbf{B} U^{n}+\Delta t \mathbf{C} V^{n}+\left(\frac{1}{2}-\beta\right) \Delta t^{2} \mathbf{B} \ddot{U}^{n}+\beta \Delta t^{2} \mathbf{B} \ddot{U}^{n+1}, \\
\mathbf{C} V^{n+1}=\mathbf{C} V^{n}+\frac{\Delta t}{2} \mathbf{B} \ddot{U}^{n}+\frac{\Delta t}{2} \mathbf{B} \ddot{U}^{n+1} .
\end{array}\right.
$$

It is well-known it is possible to derive a two-step scheme by eliminating velocity from this relations. First, we write

which leads to

$$
\left\{\begin{array}{l}
\mathbf{B} U^{n}=\mathbf{B} U^{n-1}+\Delta t \mathbf{C} V^{n-1}+\left(\frac{1}{2}-\beta\right) \Delta t^{2} \mathbf{B} \ddot{U}^{n-1}+\beta \Delta t^{2} \mathbf{B} \ddot{U}^{n}, \\
\mathbf{B} U^{n+1}=\mathbf{B} U^{n}+\Delta t \mathbf{C} V^{n}+\left(\frac{1}{2}-\beta\right) \Delta t^{2} \mathbf{B} \ddot{U}^{n}+\beta \Delta t^{2} \mathbf{B} \ddot{U}^{n+1}
\end{array}\right.
$$

$$
\begin{aligned}
\mathbf{B}\left(U^{n+1}-2 U^{n}+U^{n-1}\right)= & \Delta t \mathbf{C}\left(V^{n}-V^{n-1}\right) \\
& +\Delta t^{2} \mathbf{B}\left(\beta \ddot{U}^{n+1}+\left(\frac{1}{2}-2 \beta\right) \ddot{U}^{n}-\left(\frac{1}{2}-\beta\right) \ddot{U}^{n-1}\right),
\end{aligned}
$$

and finally, with the second equation of (3.4), written at step $n$ instead of $n+1$

$$
\mathbf{B}\left(U^{n+1}-2 U^{n}+U^{n-1}\right)=\Delta t^{2} \mathbf{B}\left(\beta \ddot{U}^{n+1}+(1-2 \beta) \ddot{U}^{n}+\beta \ddot{U}^{n-1}\right) .
$$

Multiplying this relation by $\mathbf{B}^{T} \mathbf{C}^{-1}$, we obtain

$$
\mathbf{M}\left(U^{n+1}-2 U^{n}+U^{n-1}\right)=\Delta t^{2} \mathbf{M}\left(\beta \ddot{U}^{n+1}+(1-2 \beta) \ddot{U}^{n}+\beta \ddot{U}^{n-1}\right),
$$

where $\mathbf{M}$ is the singular mass matrix. As usual for Newmark scheme, we replace acceleration by its value, given by the equilibrium equation. Hence, in the case of the singular mass matrix approach, Newmark scheme reads

$$
\mathbf{M} \frac{U^{n+1}-2 U^{n}+U^{n-1}}{\Delta t^{2}}+\mathbf{K}\left(\beta U^{n+1}+(1-2 \beta) U^{n}+\beta U^{n-1}\right)=\left(\beta \tilde{F}^{n+1}+(1-2 \beta) \tilde{F}^{n}+\beta \tilde{F}^{n-1}\right),
$$

which reads exactly as the Newmark scheme except the regular mass matrix $\mathbf{M}_{r}$ has been replaced by the singular one. 
Therefore, in the case of Newmark scheme, the singular mass matrix approach leads to a scheme similar to $(3.3)$

$$
\left\{\begin{array}{c}
\text { Find } U^{n+1, e}=\frac{U^{n+1}+e U^{n-1}}{1+e} \in \mathbb{K}^{h} \text { such that for all } W \in \mathbb{K}^{h} \\
\left(W-U^{n+1, e}\right)^{T}\left(\mathbf{M} \frac{U^{n+1}-2 U^{n}+U^{n-1}}{\Delta t^{2}}+\mathbf{K}\left(\beta U^{n+1}+(1-2 \beta) U^{n}+\beta U^{n-1}\right)\right) \\
\geq\left(W-U^{n+1, e}\right)^{T} F^{n, \beta} .
\end{array}\right.
$$

To conclude, let us remark that, as matrix $\mathbf{K}$ is positive definite, this variational inequality has always a unique solution even if $\mathbf{M}$ is singular.

Remark 3.2. As mentioned above, for $e=0$, problem (3.5) is formally identical to (3.1). The only difference lies in the expression of the mass matrix. For (3.1), Dumont-Paoli [4] established unconditional stability when $\beta=1 / 2$. As their proof doesn't use the invertibility of the mass matrix, a similar calculation should prove the same result for singular mass matrix approach for any $e$. Nevertheless, the main difficulty is to write the continuous problem associated to a singular mass operator and, in particular, to take into account that velocity is a bounded variation function, including its reliance on restitution coefficient, and acceleration is a differential measure.

Remark 3.3. Now, let us discuss very briefly strong convergence of the discrete solution. Ahn and Stewart [1] show that the amount of energy in high frequency modes, related to the fourth-order operator, is almost zero under the assumption of strong convergence to the solution of (1.3). From a mechanical point of view, it means that high frequency modes would be damped. As the singular mass matrix method transforms (1.3) into a Lipschitz-O.D.E., for a given space step, every convergent scheme for a Lipschitz-O.D.E. will converge when $\Delta t$ goes to zero, and the limit is obviously the unique solution of (2.2) which is conservative. So, if it can be proved the weak convergence of the solution of (3.5) to the problem (1.3), the strong convergence will follow from the balance of energy with the singular mass matrix (2.3). However, the same problem as in the previous remark appears. Indeed, equation (1.3) doesn't include the restitution coefficient introduced by (1.4), nor the derived Lipschitz-O.D.E. Consequently, the complete link between full discretized schemes, including restitution coefficient, and initial P.D.E. has to be investigated in depth.

\subsection{Midpoint schemes}

Following Renard [16], the midpoint scheme applied to Problem (2.2) consists in finding $U^{n+1 / 2}$ in $\mathbb{K}^{h}$ such that

$$
\left\{\begin{array}{l}
\left(W-U^{n+1 / 2}\right)^{T}\left(\mathbf{M} A^{n+1 / 2}+\mathbf{K} U^{n+1 / 2}\right) \geq\left(W-U^{n+1 / 2}\right)^{T} F^{n}, \quad \forall W \in \mathbb{K}^{h}, \\
U^{n+1 / 2}=\frac{U^{n}+U^{n+1}}{2}, \quad V^{n+1 / 2}=\frac{V^{n}+V^{n+1}}{2}, \\
\mathbf{B} U^{n+1}=\mathbf{B} U^{n}+\Delta t \mathbf{C} V^{n+1 / 2}, \quad \mathbf{C} V^{n+1}=\mathbf{C} V^{n}+\Delta t \mathbf{B} A^{n+1 / 2},
\end{array}\right.
$$

where $A^{n+1 / 2}$ is acceleration at "middle time step" $n+1 / 2$. As matrix $\mathbf{C}$ is invertible, we have

$$
V^{n+1}=2 V^{n+1 / 2}-V^{n}=2 \mathbf{C}^{-1} \mathbf{B} \frac{U^{n+1}-U^{n}}{\Delta t}-V^{n}=4 \mathbf{C}^{-1} \mathbf{B} \frac{U^{n+1 / 2}-U^{n}}{\Delta t}-V^{n} .
$$

Moreover, $A^{n+1 / 2}$ can be eliminated in the following way

$$
\mathbf{M} A^{n+1 / 2}=\mathbf{B}^{T} \mathbf{C}^{-1} \mathbf{B} A^{n+1 / 2}=\mathbf{B}^{T} \mathbf{C}^{-1} \frac{\mathbf{C} V^{n+1}-\mathbf{C} V^{n}}{\Delta t}=\mathbf{B}^{T} \frac{V^{n+1}-V^{n}}{\Delta t}
$$

or more explicitly

$$
\mathbf{M} A^{n+1 / 2}=4 \mathbf{B}^{T} \mathbf{C}^{-1} \mathbf{B} \frac{U^{n+1 / 2}-U^{n}}{\Delta t^{2}}-2 \mathbf{B}^{T} \frac{V^{n}}{\Delta t}=\frac{4}{\Delta t^{2}} \mathbf{M} U^{n+1 / 2}-\frac{4}{\Delta t^{2}} \mathbf{M} U^{n}-\frac{2}{\Delta t} \mathbf{B}^{T} V^{n} .
$$


Then, a new formulation of (3.6) is

$$
\left\{\begin{array}{l}
U^{n} \text { and } V^{n} \text { being given, find } U^{n+1 / 2} \in \mathbb{K}^{h} \text { such that } \\
\left(W-U^{n+1 / 2}\right)^{T}\left(\frac{4}{\Delta t^{2}} \mathbf{M} U^{n+1 / 2}+\mathbf{K} U^{n+1 / 2}\right) \geq\left(W-U^{n+1 / 2}\right)^{T} \bar{F}^{n}, \quad \forall W \in \mathbb{K}^{h}, \\
\text { where } \bar{F}^{n}=F^{n}+\frac{4}{\Delta t^{2}} \mathbf{M} U^{n}+\frac{2}{\Delta t} \mathbf{B}^{T} V^{n} \\
U^{n+1}=2 U^{n+1 / 2}-U^{n}, \quad V^{n+1}=2 \mathbf{C}^{-1} \mathbf{B} \frac{U^{n+1}-U^{n}}{\Delta t}-V^{n} .
\end{array}\right.
$$

Exactly as for Newmark scheme, this variational inequality has always a unique solution even if $\mathbf{M}$ is singular.

Let us observe that midpoint scheme corresponds to $e=1$, which is a perfect elastic impact. In fact, when a shock occurs, $U^{n+1 / 2}$ belongs to the boundary of $\mathbb{K}^{h}$. At this moment, it means the velocity $V^{n+1 / 2}=0$ and then $V^{n+1}=-V^{n}$ : velocity just after the impact is the same, up to its sign, than before. Consequently, a way to introduce the restitution coefficient in midpoint scheme is to set

$$
V^{n+1 / 2}=\left\{\begin{array}{ll}
\frac{V^{n}+V^{n+1}}{2} & \text { when there is no shock, } \\
\frac{e V^{n}+V^{n+1}}{1+e} & \text { if shock occurs, }
\end{array}\right\}=\frac{\xi V^{n}+V^{n+1}}{1+\xi}
$$

where $\xi=1$ or $\xi=e$ depending on whether there is a shock or not. Then, (3.7) has to be modified in the appropriate way.

Remark 3.4. In the case $\mathbb{H}^{h}=\mathbb{W}^{h}$, which corresponds to the classical discretization where displacement and velocity are approximated in the same way, we obtain a scheme similar to (3.7), the only differences being the singular mass matrix $\mathbf{M}$ is replaced by the regular one $\mathbf{M}_{r}$ and $\mathbf{C}=\mathbf{B}=\mathbf{B}^{T}$ so $\mathbf{C}^{-1} \mathbf{B}=I$. Finally, we do not prove the convergence of these schemes towards a solution of the continuous problem. Nevertheless, we shall observe good numerical results with singular mass discretization and instabilities for regular one when $e=1$.

\section{Numerical RESUlts}

As in Dumont-Paoli [4], it is considered the case of a steel pipe, which length is $L=1.501 \mathrm{~m}$, external diameter is equal to $1 \mathrm{~cm}$ and thickness is $0.5 \mathrm{~mm}$. The material properties are characterized by its Young modulus $E=2 \times 10^{11} \mathrm{~Pa}$ and its density $\rho=8.10^{3} \mathrm{~kg} / \mathrm{m}^{3}$. Thus, in this case, we have $\frac{E I}{\rho S}=282.84 \mathrm{~m}^{4} \mathrm{~s}^{-2}$, where $I$ is the quadratic momentum of inertia of the beam and $S$ its section. All the next computations will use Matlab.

Moreover, in the following, we will consider two kinds of obstacles (see (2.4) for definition of convex $\mathbb{K}^{h}$ ):

- Punctual stops at the right edge of the beam

$$
\left\{\begin{array}{l}
g_{2}(L)=-g_{1}(L)=0.1, \\
g_{2}(x)=-g_{1}(x)=+\infty, \quad \forall x \in[0, L[
\end{array}\right.
$$

- Flat obstacles all along the beam

$$
g_{2}(x)=-g_{1}(x)=0.1, \quad \forall x \in[0, L] .
$$




\subsection{Some aspects of numerical implementation}

To explain how the algorithm works in the general case, let us begin with the fully implicit Newmark-DumontPaoli scheme (3.1). First, it can be rewritten as

$$
\left\{\begin{array}{l}
\text { Find } U^{n+1} \in \mathbb{K}^{h} \text { such that for all } W \in \mathbb{K}^{h} \\
\left(W-U^{n+1}\right)^{T}\left(\frac{\mathbf{M}_{r}}{\Delta t^{2}} U^{n+1}+\beta \mathbf{K} U^{n+1}\right) \geq\left(W-U^{n+1}\right)^{T} \tilde{F}^{n}
\end{array}\right.
$$

where

$$
\tilde{F}^{n}=F^{n, \beta}-\mathbf{M}_{r} \frac{-2 U^{n}+U^{n-1}}{\Delta t^{2}}-\mathbf{K}\left((1-2 \beta) U^{n}+\beta U^{n-1}\right),
$$

$F^{n, \beta}$ being given by (3.2). Since matrix $\mathbf{A} \equiv \frac{1}{\Delta t^{2}} \mathbf{M}_{r}+\beta \mathbf{K}$ is positive definite and symmetric, equation (4.2) is equivalent to the following minimization problem

$$
U^{n+1}=\operatorname{Argmin}_{W \in \mathbb{K}^{h}}\left(\frac{1}{2} W^{T} \mathbf{A} W-\left(\tilde{F}^{n}\right)^{T} W\right) .
$$

Let us remark that, as constraints defining $\mathbb{K}^{h}$ are linear, the above minimization problem is a linear quadratic problem, which can be solved by different methods. As in [5], we will use the Matlab function "quadprog", which uses a Lagrange multipliers method. Nevertheless, in the case of punctual obstacles (4.1), we will directly compute $U^{n+1}$ as the projection of the unconstrained solution on $\mathbb{K}^{h}$, which is much more efficient than "quadprog" function.

Now, let us study the general case $(e \neq 0)$, corresponding to $(3.3)$, we recall here

$$
\left\{\begin{array}{c}
\text { Find } U^{n+1, e}=\frac{U^{n+1}+e U^{n-1}}{1+e} \in \mathbb{K}^{h} \text { such that for all } W \in \mathbb{K}^{h} \\
\left(W-U^{n+1, e}\right)^{T}\left(\mathbf{M}_{r} \frac{U^{n+1}-2 U^{n}+U^{n-1}}{\Delta t^{2}}+\mathbf{K}\left(\beta U^{n+1}+(1-2 \beta) U^{n}+\beta U^{n-1}\right)\right) \\
\geq\left(W-U^{n+1, e}\right)^{T} F^{n, \beta}
\end{array}\right.
$$

Then, it is possible to express $U^{n+1}$ as $U^{n+1}=(1+e) U^{n+1, e}-e U^{n-1}$ and to replace it in the previous inequality

$$
\left\{\begin{array}{l}
\left(W-U^{n+1, e}\right)^{T}\left(\mathbf{M}_{r} \frac{(1+e) U^{n+1, e}-2 U^{n}+(1-e) U^{n-1}}{\Delta t^{2}}\right. \\
\left.\quad+\mathbf{K}\left(\beta(1+e) U^{n+1, e}+(1-2 \beta) U^{n}+\beta(1-e) U^{n-1}\right)\right) \geq\left(W-U^{n+1, e}\right)^{T} F^{n, \beta}
\end{array}\right.
$$

and the problem becomes

$$
\left\{\begin{array}{l}
\text { Find } U^{n+1, e} \in \mathbb{K}^{h} \text { such that for all } W \in \mathbb{K}^{h} \\
\left(W-U^{n+1, e}\right)^{T}\left(\frac{\mathbf{M}_{r}}{\Delta t^{2}} U^{n+1, e}+\beta \mathbf{K} U^{n+1, e}\right) \geq\left(W-U^{n+1, e}\right)^{T} G^{n}
\end{array}\right.
$$




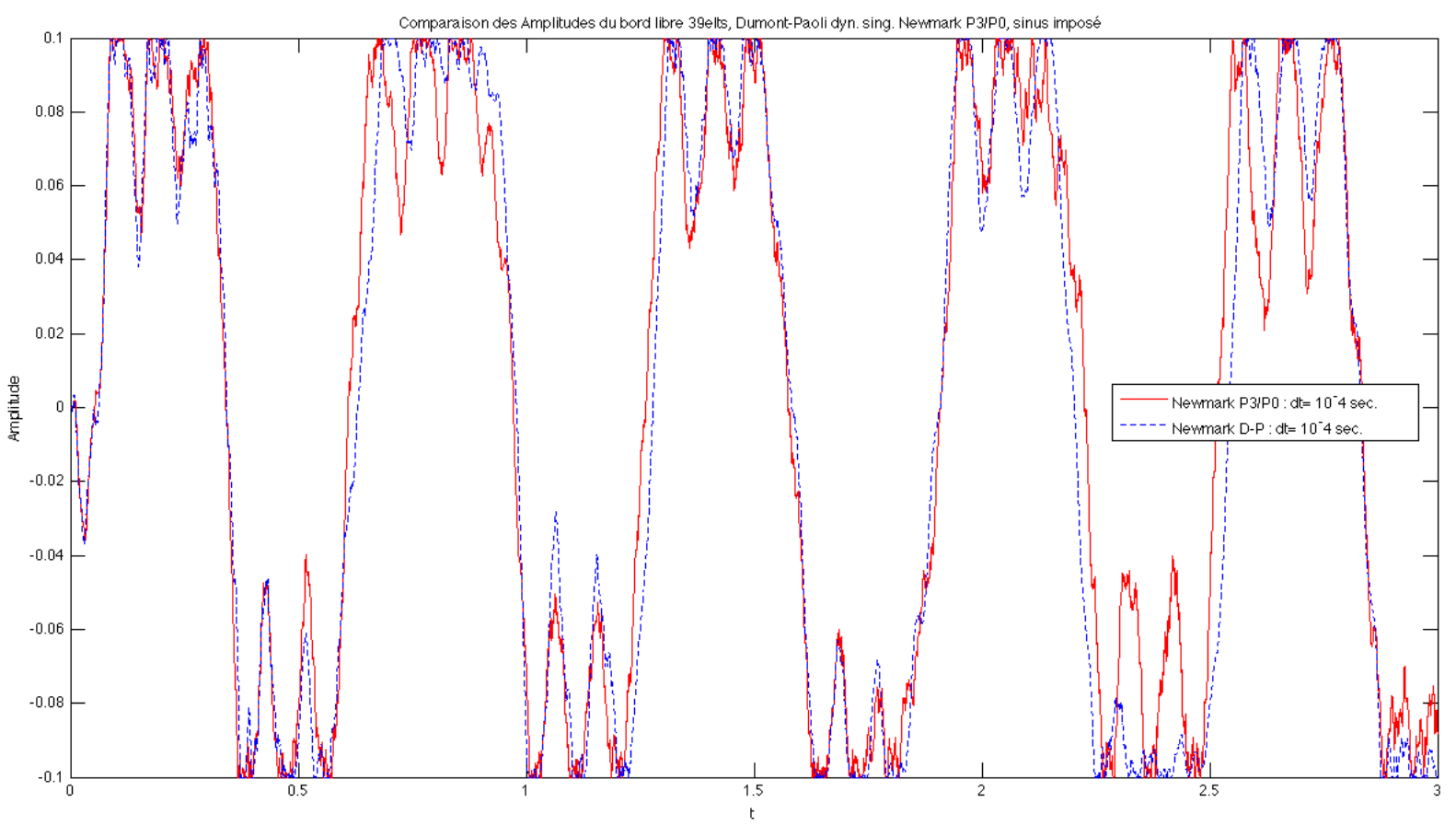

Figure 1. Regular versus P3/P0 singular mass matrix. NDP scheme. $e=0, \Delta t=10^{-4}, 39$ elements.

where

$$
G^{n}=\frac{1}{1+e}\left(\tilde{F}^{n}+e\left(\frac{1}{\Delta t^{2}} \mathbf{M}_{r}+\beta \mathbf{K}\right) U^{n-1}\right) .
$$

It looks like exactly as (4.2) and is solved in the same way.

Consequently, the way to solve (3.3) follows Paoli-Schatzman [13]. First, we calculate $Q^{n+1} \equiv \mathbf{A}^{-1} \tilde{F}^{n}$. Second, the following alternative holds:

- if $\frac{Q^{n+1}+e U^{n-1}}{1+e}$ belongs to $\mathbb{K}^{h}$, equation (4.3) is verified and we set $U^{n+1}=Q^{n+1}$;

- if $\frac{Q^{n+1}+e U^{n-1}}{1+e}$ doesn't belong to $\mathbb{K}^{h}$, we solve (4.3) with "quadprog" function or direct projection, and we set $U^{n+1}=(1+e) U^{n+1, e}-e U^{n-1}$.

It has to be said that the fact $U^{n+1, e}$ belongs to $\mathbb{K}^{h}$ doesn't mean that $U^{n+1}$ belongs to $\mathbb{K}^{h}$ too. By the way, it doesn't but it is close. This phenomenon is also pointed out by Paoli-Schatzman [13].

Remark 4.1. The case of a singular mass matrix (Eq. (3.5)) is handled exactly in the same way as matrix $\frac{1}{\Delta t^{2}} \mathbf{M}+\beta \mathbf{K}$ has the same properties than the above defined matrix $\mathbf{A}$.

\subsection{Case of pointwise stops with $e=0$}

In this section, we consider the fully implicit Newmark-Dumont-Paoli (NDP) scheme and we compare the regular mass matrix discretization (3.1) with the singular ones (3.5). So we use the two kinds of singular mass matrices $\left(P_{3} / P_{0}\right.$ and $\left.P_{3} / P_{1}\right)$, as they are described in the previous sections. Then, the next figures give, for various time steps, the numerical displacement of the right edge of the beam. For all Newmark schemes, parameter $\beta$ is taken equal to $1 / 2$, which insures unconditional stability. Finally, initial conditions correspond to a sine-sweep base forced vibration (1.2) of amplitude $a=0.2$ and frequency $\omega=10 \mathrm{~Hz}$. 


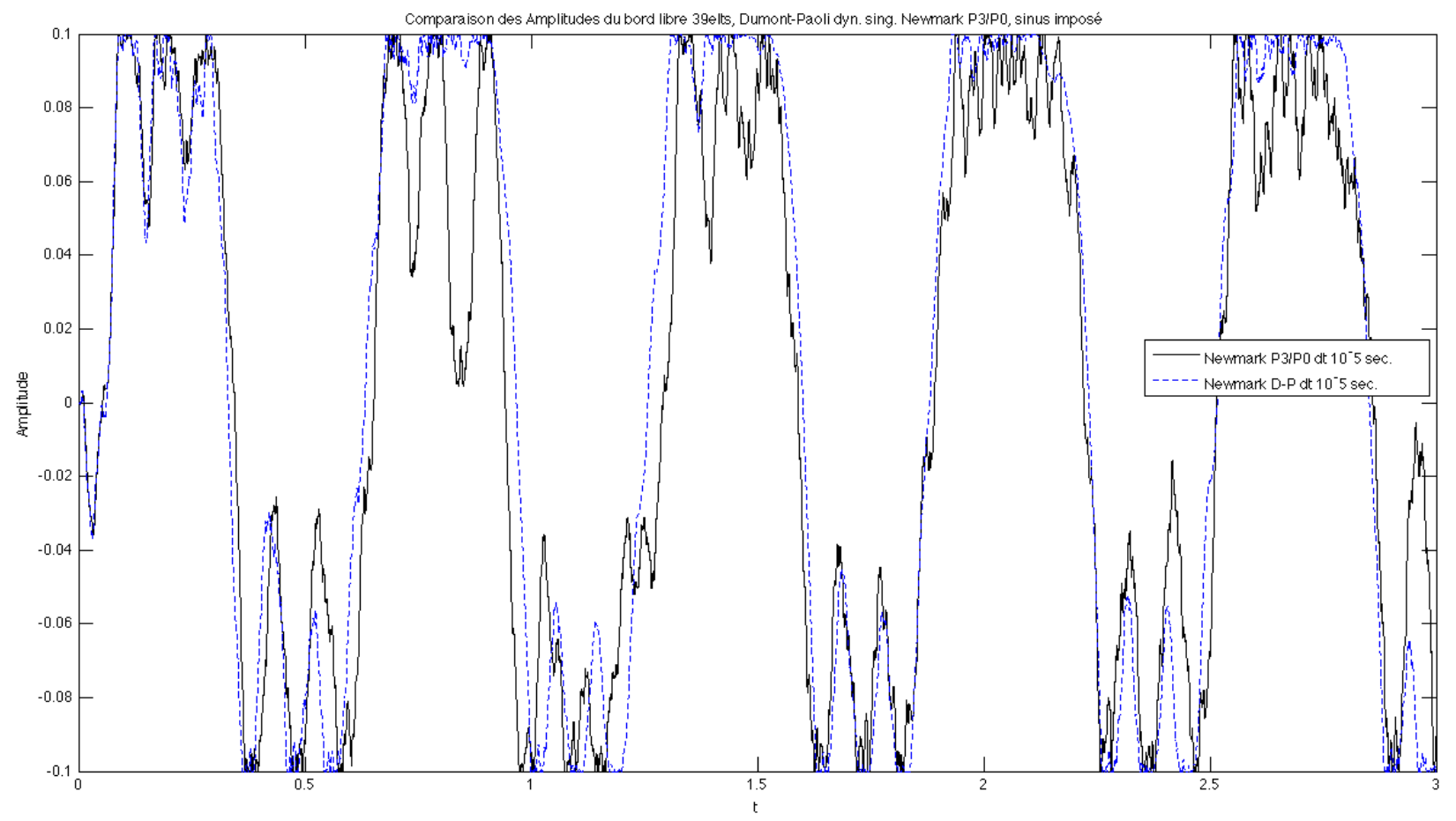

Figure 2. Regular versus $\mathrm{P} 3 / \mathrm{P} 0$ singular mass matrix. NDP scheme. $e=0, \Delta t=10^{-5}, 39$ elements.

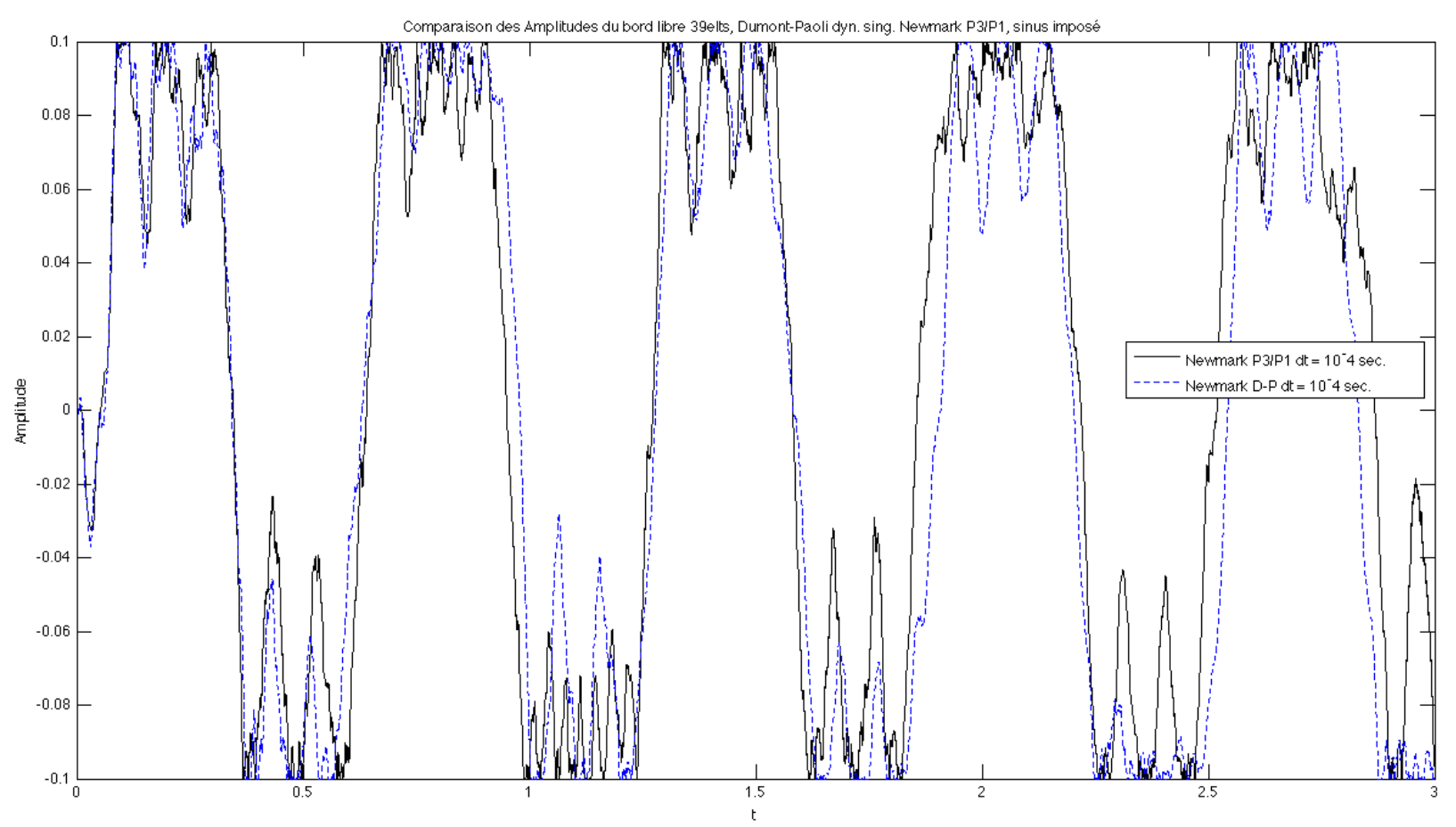

Figure 3. Regular versus $\mathrm{P} 3 / \mathrm{P} 1$ singular mass matrix. NDP scheme. $e=0, \Delta t=10^{-4}, 39$ elements. 


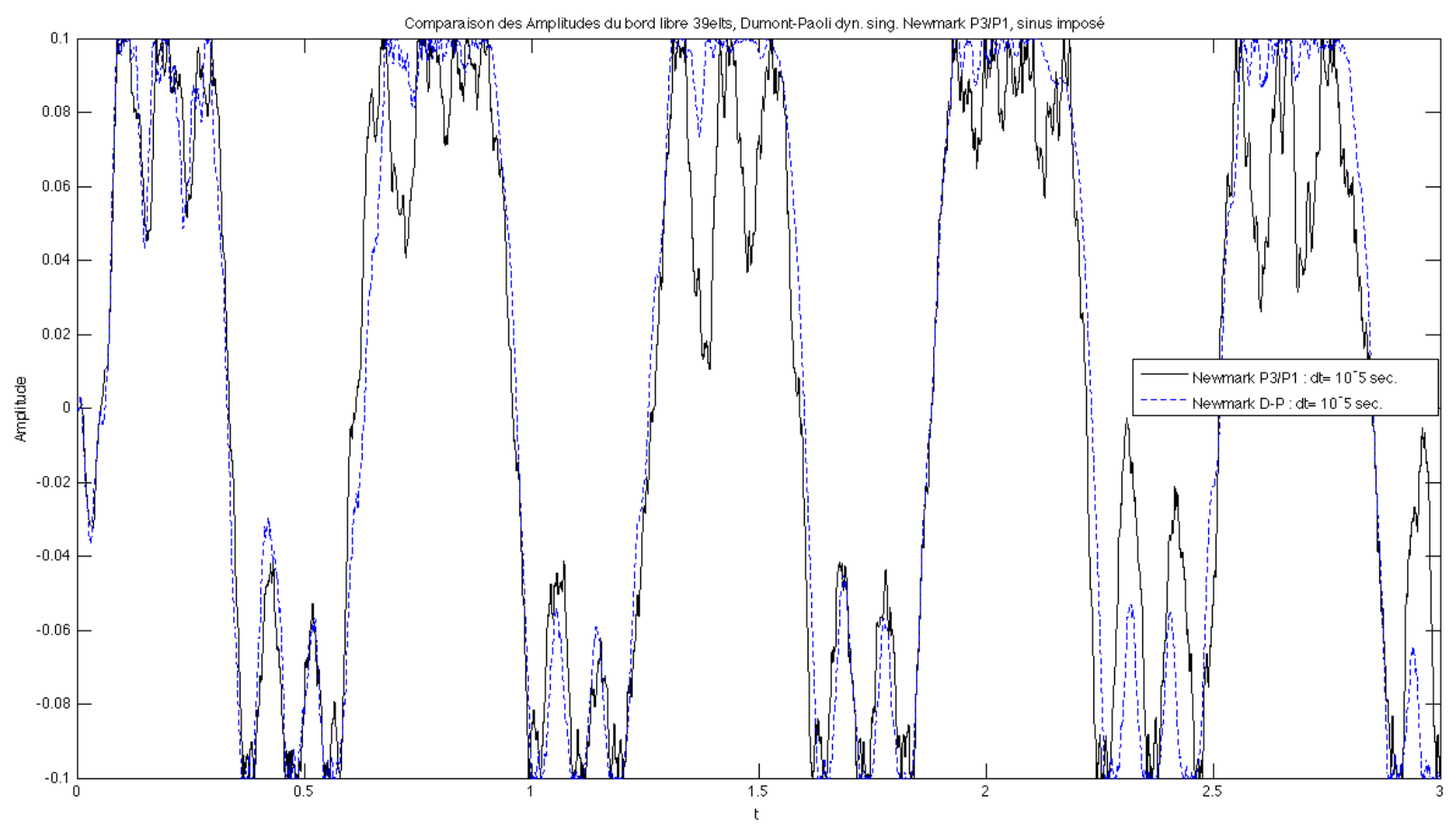

FigurE 4. Regular versus $\mathrm{P} 3 / \mathrm{P} 1$ singular mass matrix. NDP scheme. $e=0, \Delta t=10^{-5}$, 39 elements.
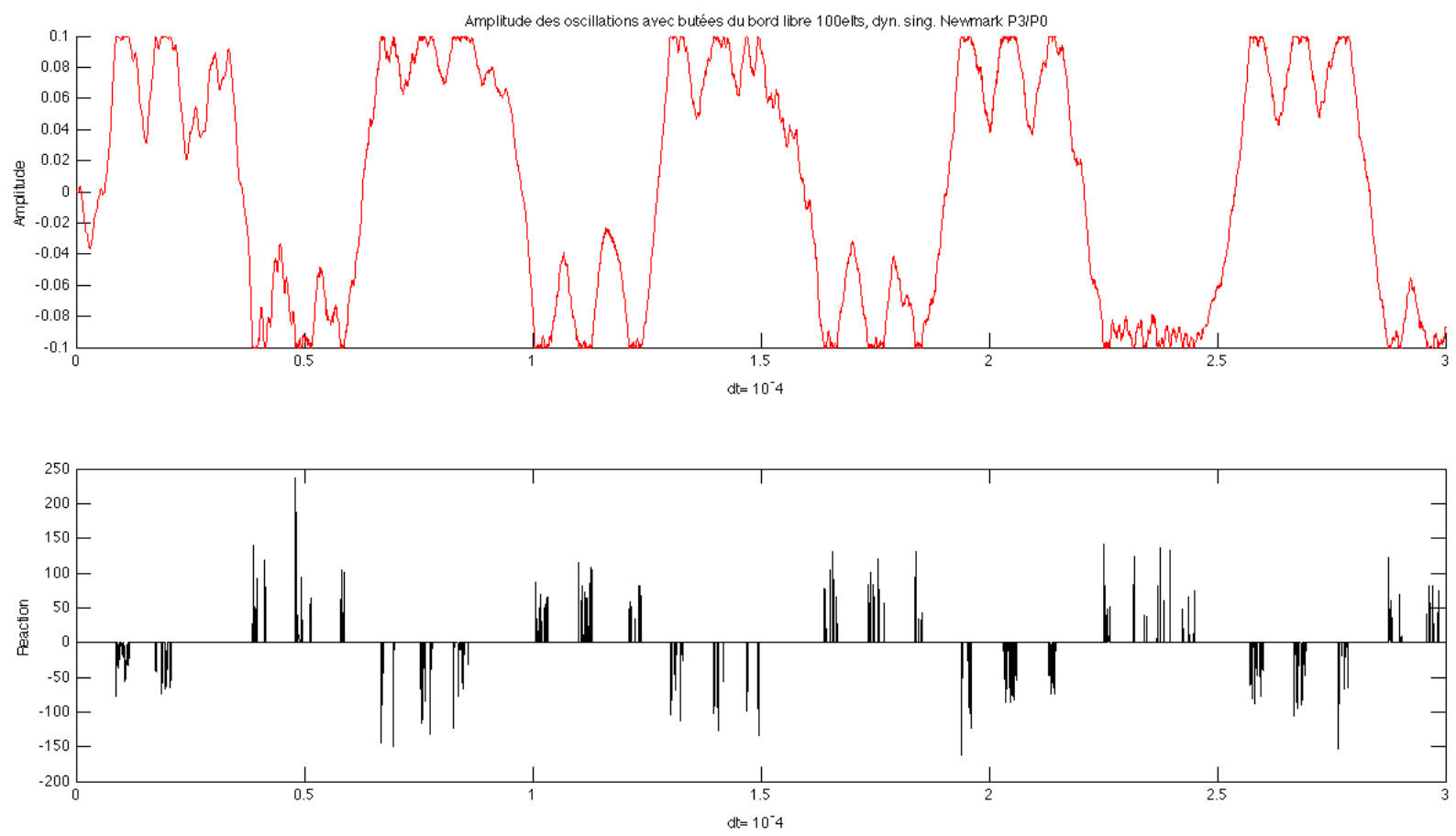

FIgURE 5. Displacement and reaction with NDP P3/P0 singular mass matrix. $e=0, \Delta t=$ $10^{-4}, 100$ elements. 

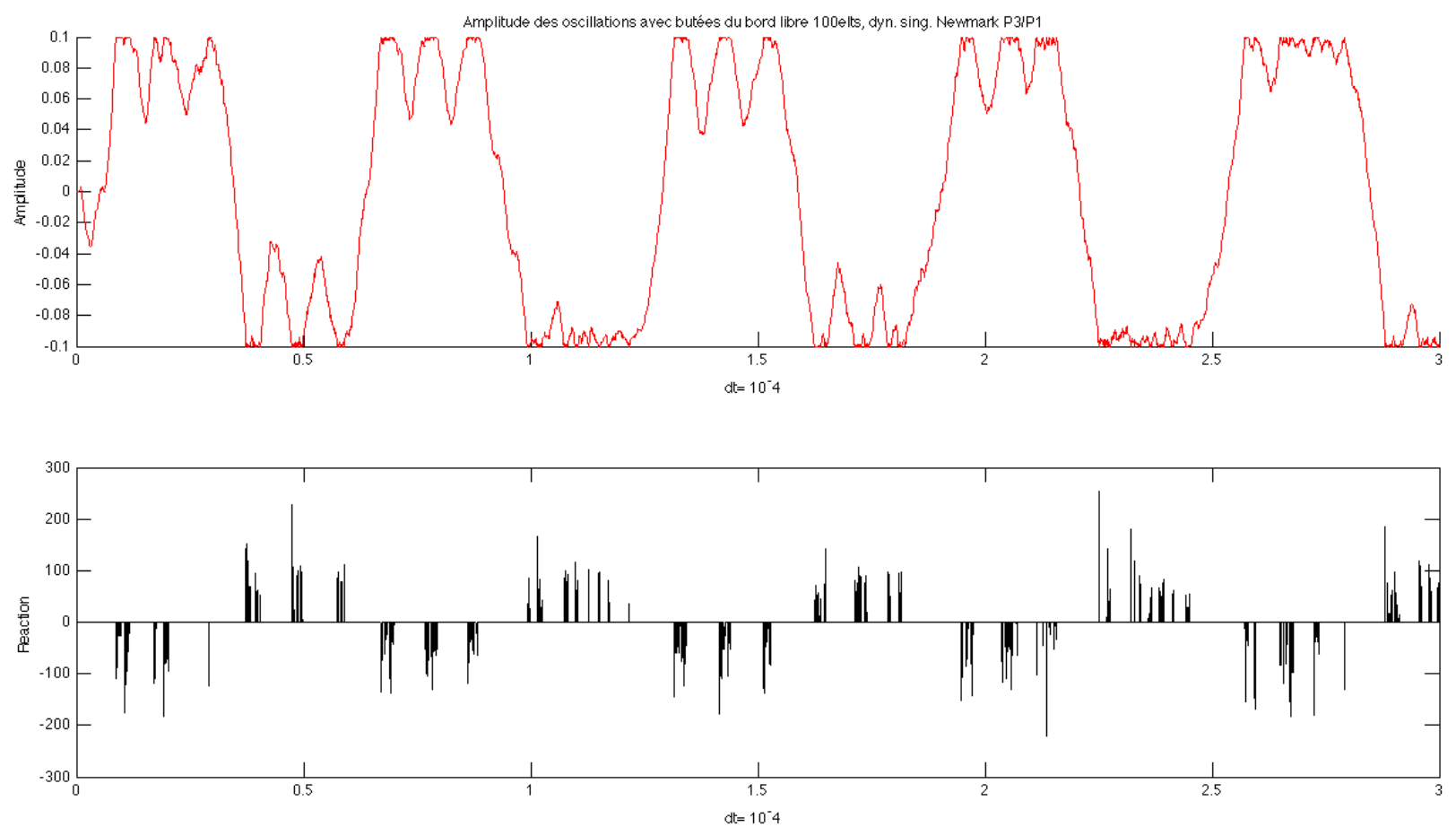

Figure 6. Displacement and reaction with NDP P3/P1 singular mass matrix. $e=0, \Delta t=$ $10^{-4}, 100$ elements.

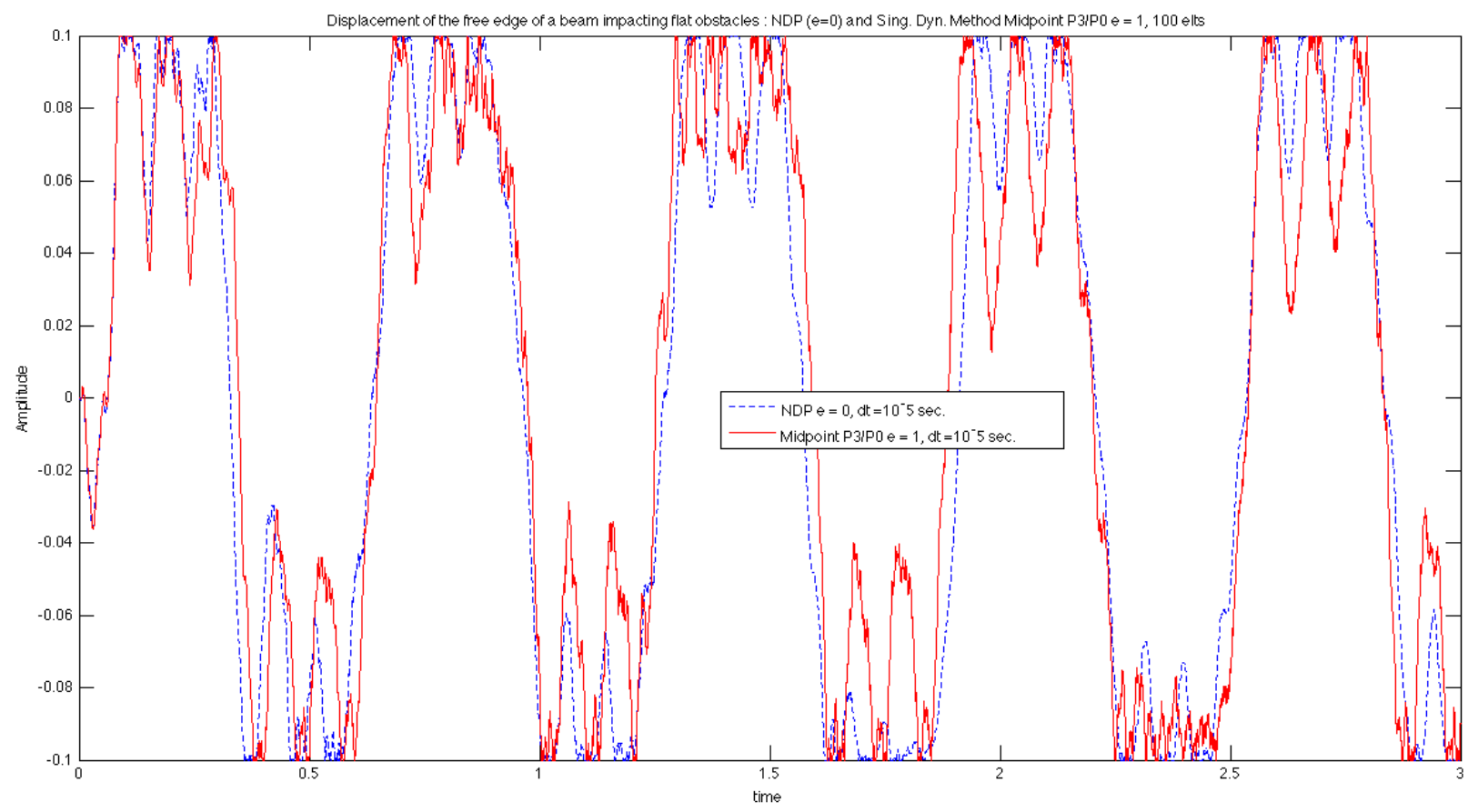

FiguRE 7. Midpoint $\mathrm{P} 3 / \mathrm{P} 0$ singular mass matrix $(e=1)$ versus NDP regular mass matrix $(e=0) . \Delta t=10^{-5}, 100$ elements. 


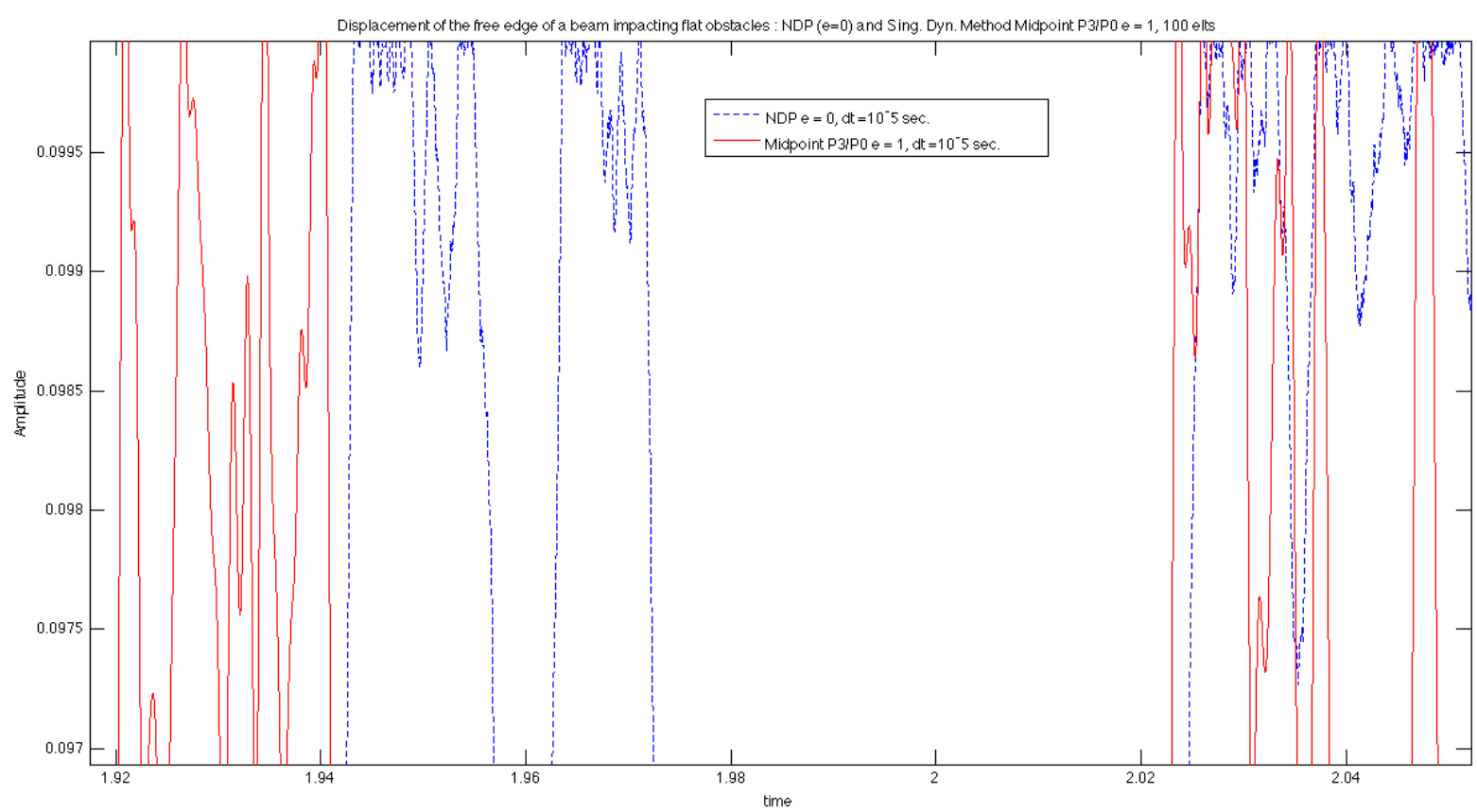

Figure 8. Zoom - Midpoint P3/P0 singular mass matrix $(e=1)$ versus NDP regular mass matrix $(e=0) . \Delta t=10^{-5}, 100$ elements.

\subsection{Case of pointwise stops with $e=0$ or $e=1$}

Here, the midpoint scheme with singular mass matrix (3.7) is compared with fully implicit NDP with regular mass matrix (3.1). By the way, the former scheme correspond to a perfect elastic impact and the latter to an absorbing one. The next figures give also the numerical displacement of the right edge of the beam, for the same initial conditions as in the previous section. As expected, it can be observed bounces after impact are generaly more important with midpoint than with NDP scheme (see zooms in Figs. 8 and 10).

\subsection{Case of flat obstacles}

Now, the case of a beam between two symmetric flat obstacles is considered. First, we study the effect of mass matrix discretization on NDP scheme. All the figures of this section give the numerical displacement of the right edge of the beam, for the same initial conditions as above. The results are very similar to those of pointwise stops. The only point to underline is the great sensitivity of the algorithm to the use of "quadprog" Matlab function (choice of parameters, initialization).

The following numerical tests show the effect of restitution coefficient $e$, which is similar to what was observed with pointwise obstacles.

\subsection{Energy conservation}

In order to study conservation of energy in the previous numerical schemes, in all the next paragraphs, we consider the case where there is no loading $f(x, t) \equiv 0$ for all $x$ and $t$. All energy is contained in the initial data, which read

$$
u_{0}(x)=0.04 x^{2}, \quad v_{0}(x)=-2 x, \quad \forall x \in[0, L] .
$$




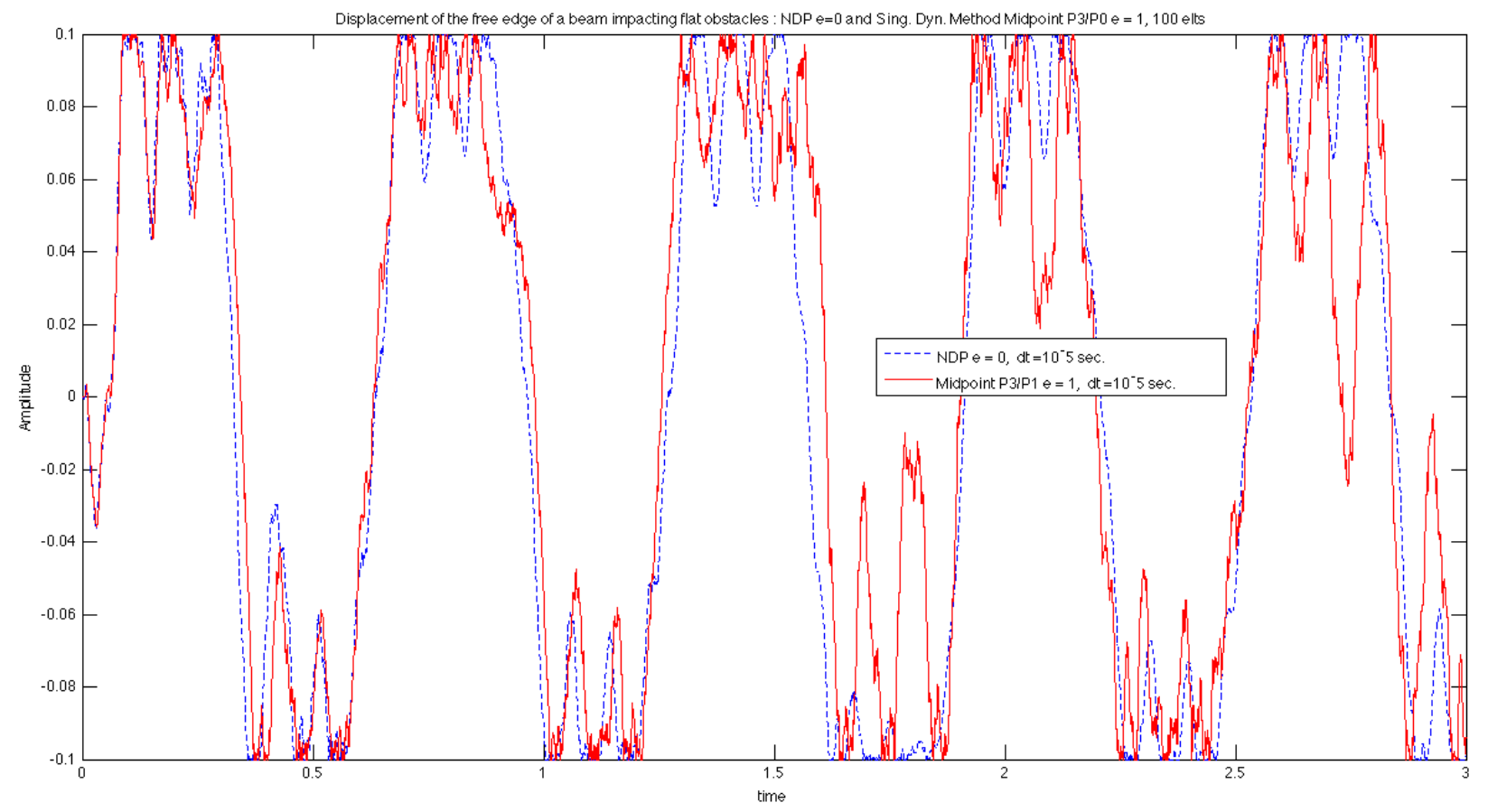

FiguRE 9. Midpoint P3/P1 singular mass matrix $(e=1)$ versus NDP regular mass matrix $(e=0) . \Delta t=10^{-5}, 100$ elements.

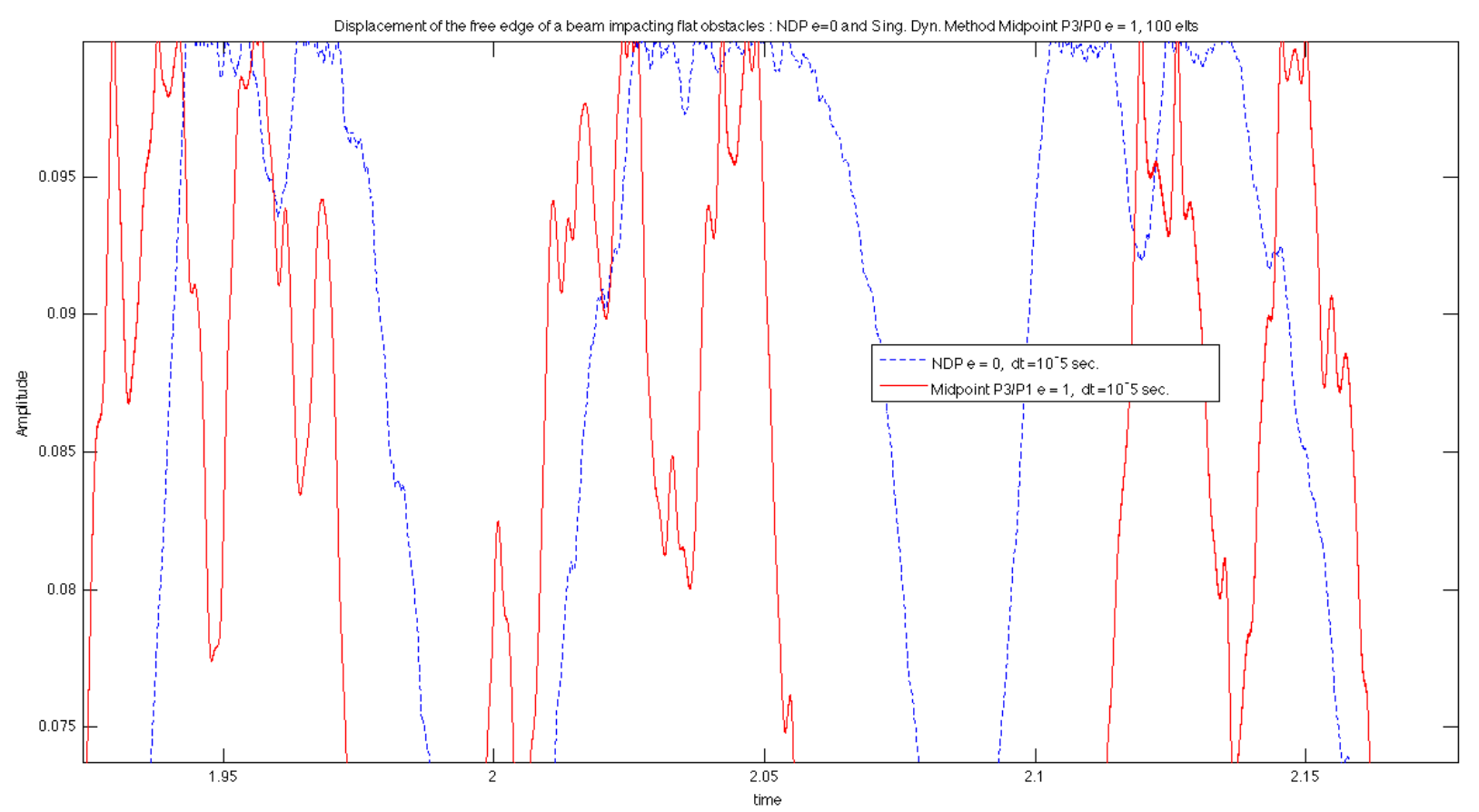

Figure 10. Zoom - Midpoint P3/P1 singular mass matrix $(e=1)$ versus NDP regular mass matrix $(e=0) . \Delta t=10^{-5}, 100$ elements. 


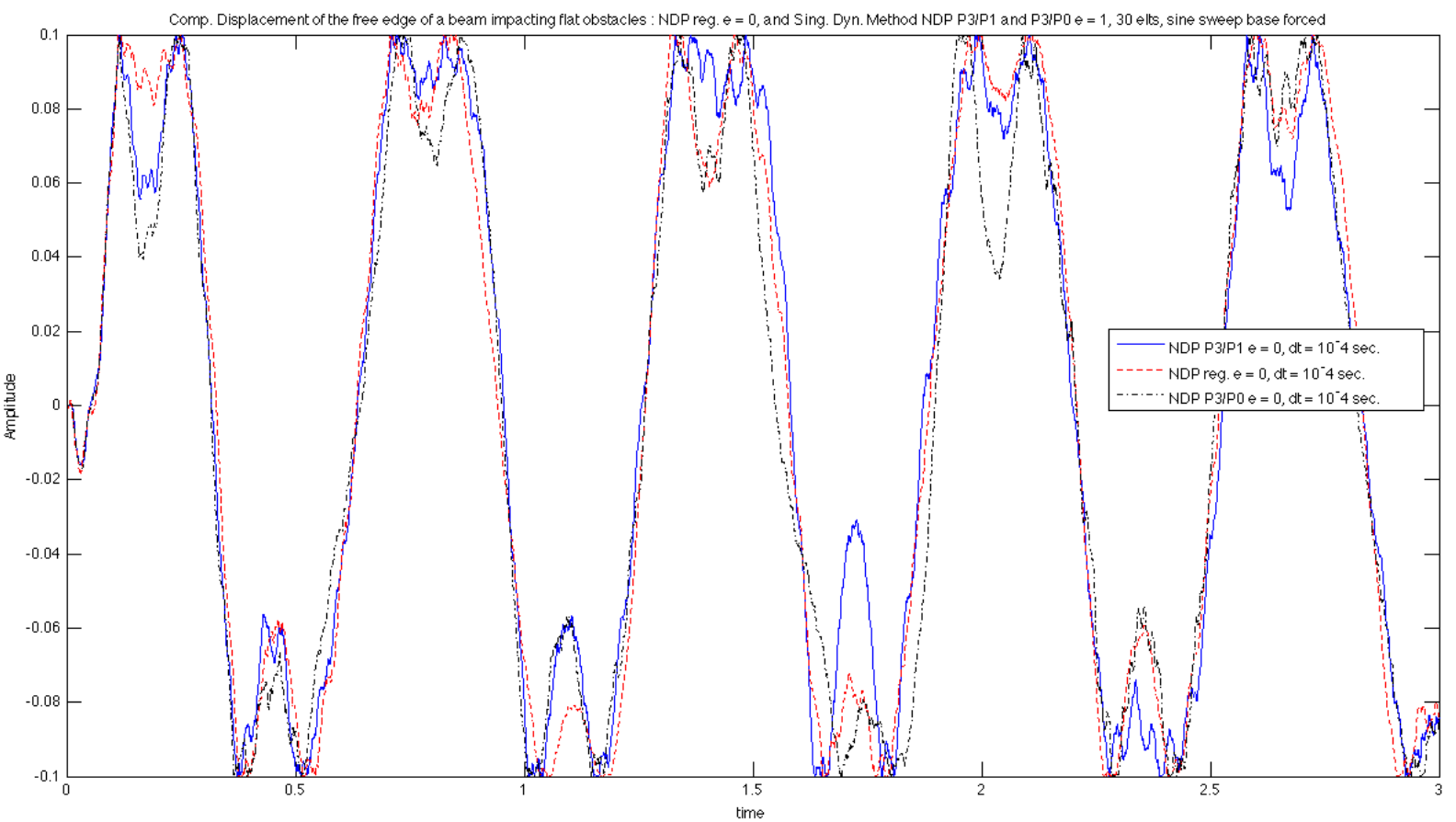

Figure 11. Regular versus $\mathrm{P} 3 / \mathrm{P} 0$ and $\mathrm{P} 3 / \mathrm{P} 1$ singular mass matrix. NDP scheme. $e=0, \Delta t=$ $10^{-4}, 30$ elements.

In the following computations, for Newmark schemes, parameter $\beta$ is chosen equal to $1 / 2$, which leads to unconditional stability. Finally, the discrete energy $E_{n}$ is given by

$$
E_{n}=\frac{1}{8 \Delta t^{2}}\left(U^{n+1}-U^{n-1}\right)^{T} \mathbf{M}\left(U^{n+1}-U^{n-1}\right)+\frac{1}{2}\left(U^{n}\right)^{T} \mathbf{K}\left(U^{n}\right),
$$

as there is no loading.

\subsubsection{Case of pointwise stops with $e=0$}

In the following figures are given the energy evolutions for different time and space steps, in the frame of Newmark-Dumont-Paoli scheme, with $\beta=1 / 2$. As the restitution coefficient $e$ is zero, this scheme is known to be dissipative [4]. In fact, we can observe the energy remains constant between two successive impacts and is decreasing only at these impacts. Nevertheless, when the time step goes to zero, the loss of energy is weaker and weaker and it can be expected that energy conservation will hold at the limit.

The two next figures allow to compare the effect of mass discretization. They show that, with singular mass matrix and for similar time steps, the loss of energy is much lower than with a regular one.

\subsubsection{Case of pointwise stops with various values of $e$}

In this section, evolution of energy is investigated for different values of the restitution coefficient $e$. First, we observe the effect of the singular mass matrix discretization for $e=1$, which corresponds to the case where energy should remain constant. The figure shows that the regular mass matrix Newmark-Dumont-Paoli scheme creates energy, so seems to strive for unstability. This effect decreases with time step. Conversely, singular mass matrix schemes keep constant energy. Moreover, as far as midpoint scheme is considered, it is unstable with regular mass matrix whereas it becomes stable and energy conservative with singular mass matrix (see Fig. 18). That's why it is compared with NDP scheme in the following figures. 


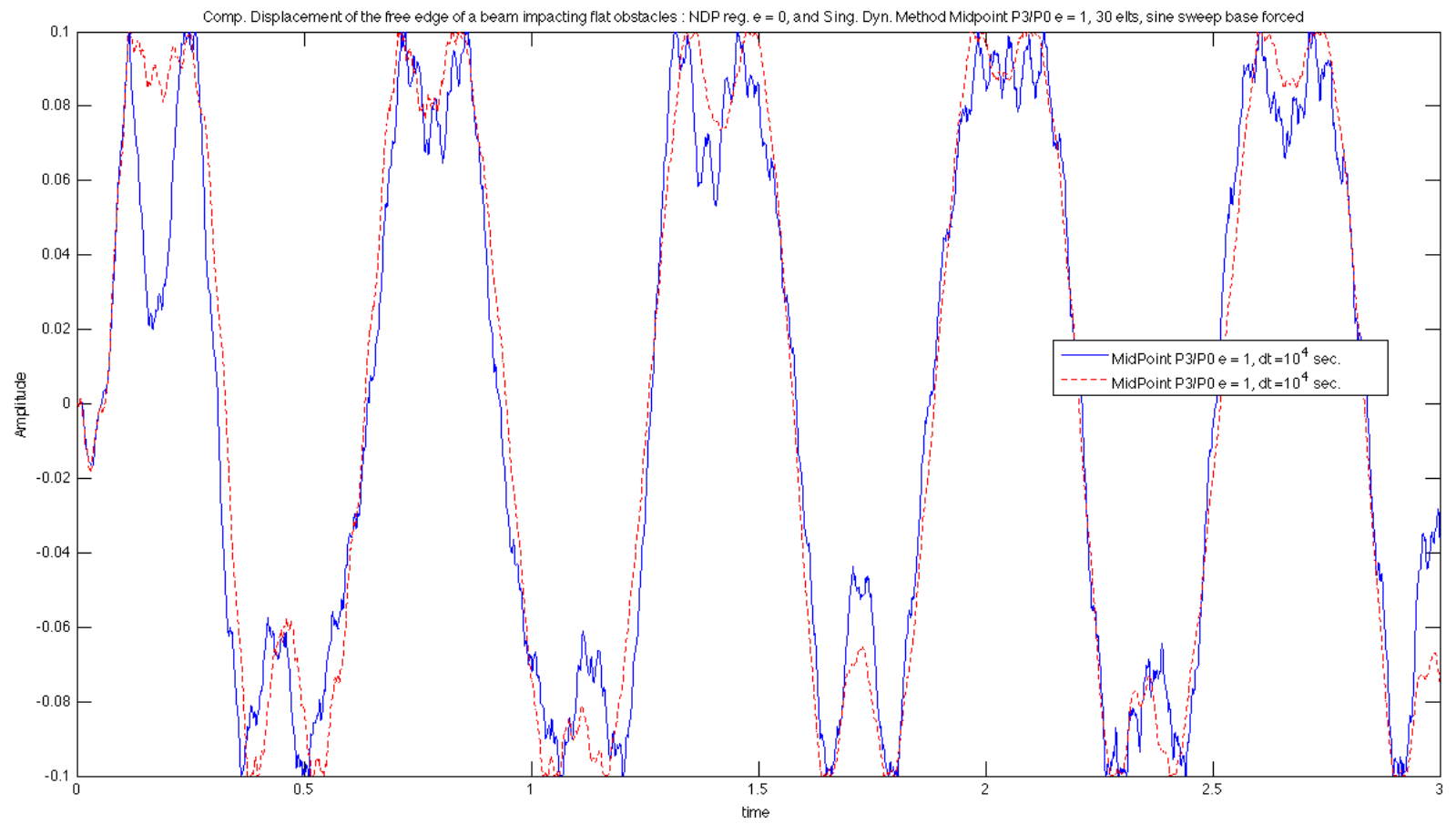

Figure 12. Midpoint P3/P0 singular mass matrix $(e=1)$ versus NDP regular mass matrix. $\Delta t=10^{-4}, 30$ elements.

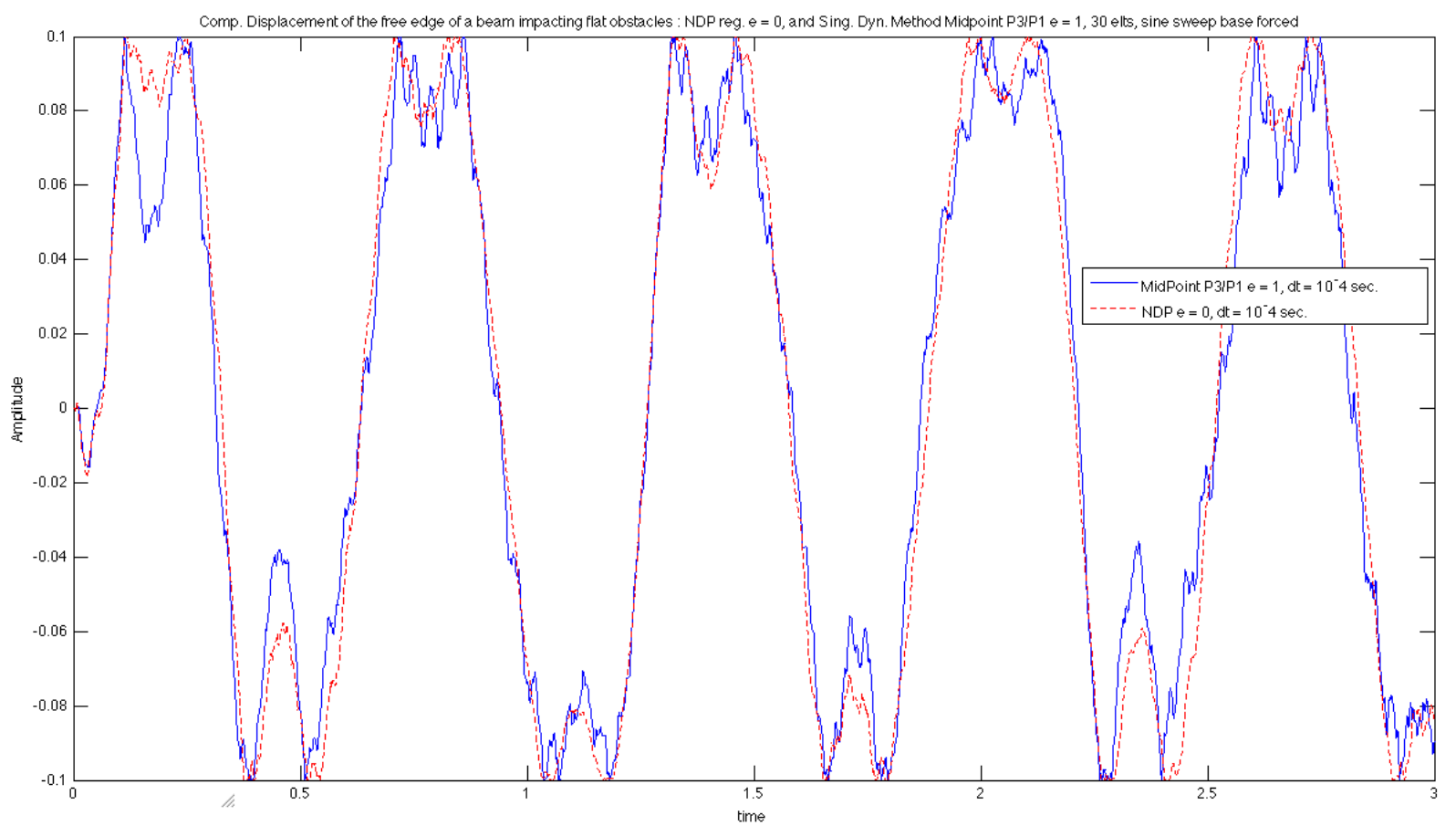

Figure 13. Midpoint P3/P1 singular mass matrix $(e=1)$ versus NDP regular mass matrix. $\Delta t=10^{-4}, 30$ elements. 


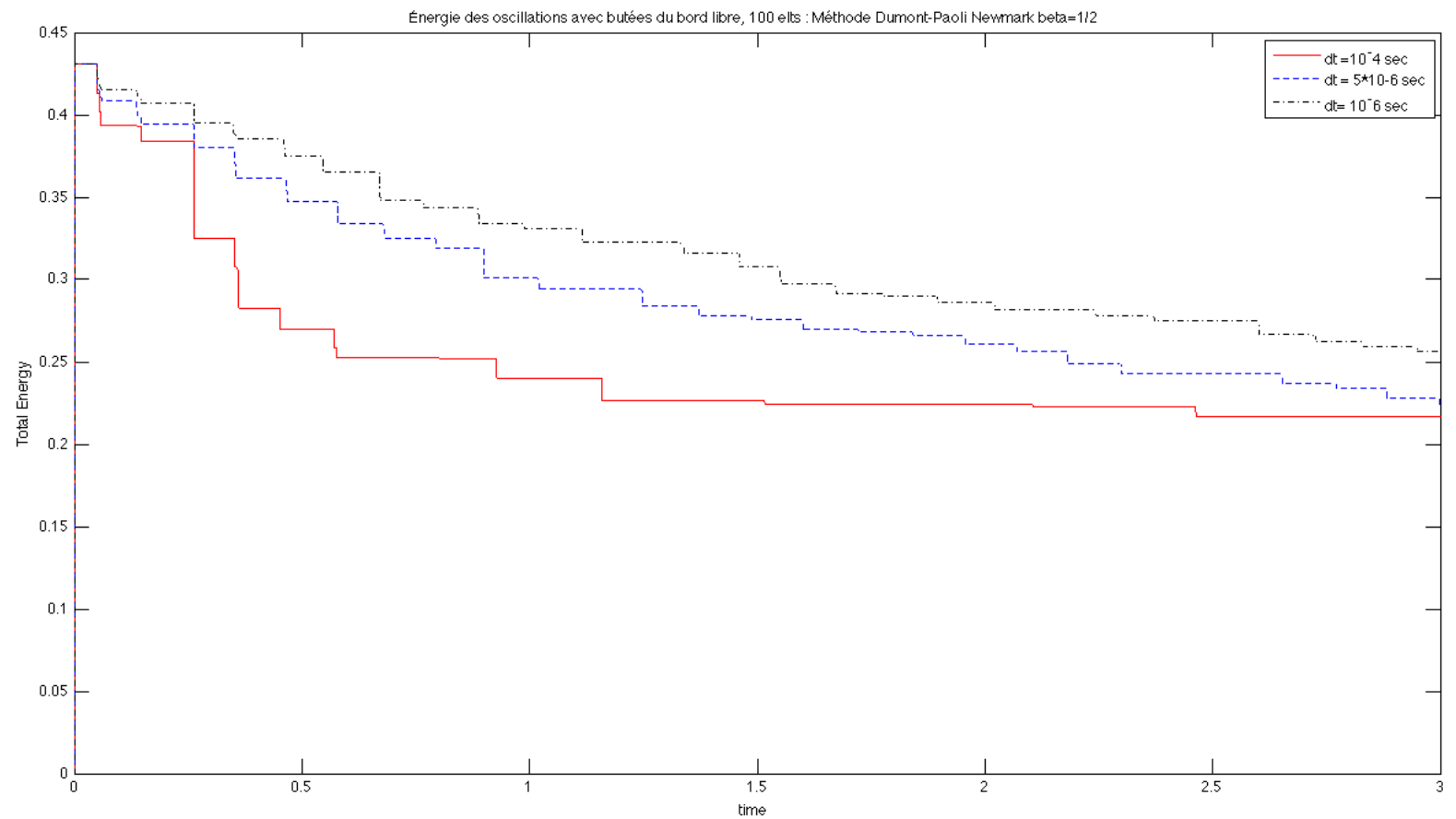

FiguRE 14. Energy for different time steps - NDP scheme with regular mass matrix. $e=$ $0, \Delta t=10^{-4}, 5.10^{-6}, 10^{-6}, 100$ elements.

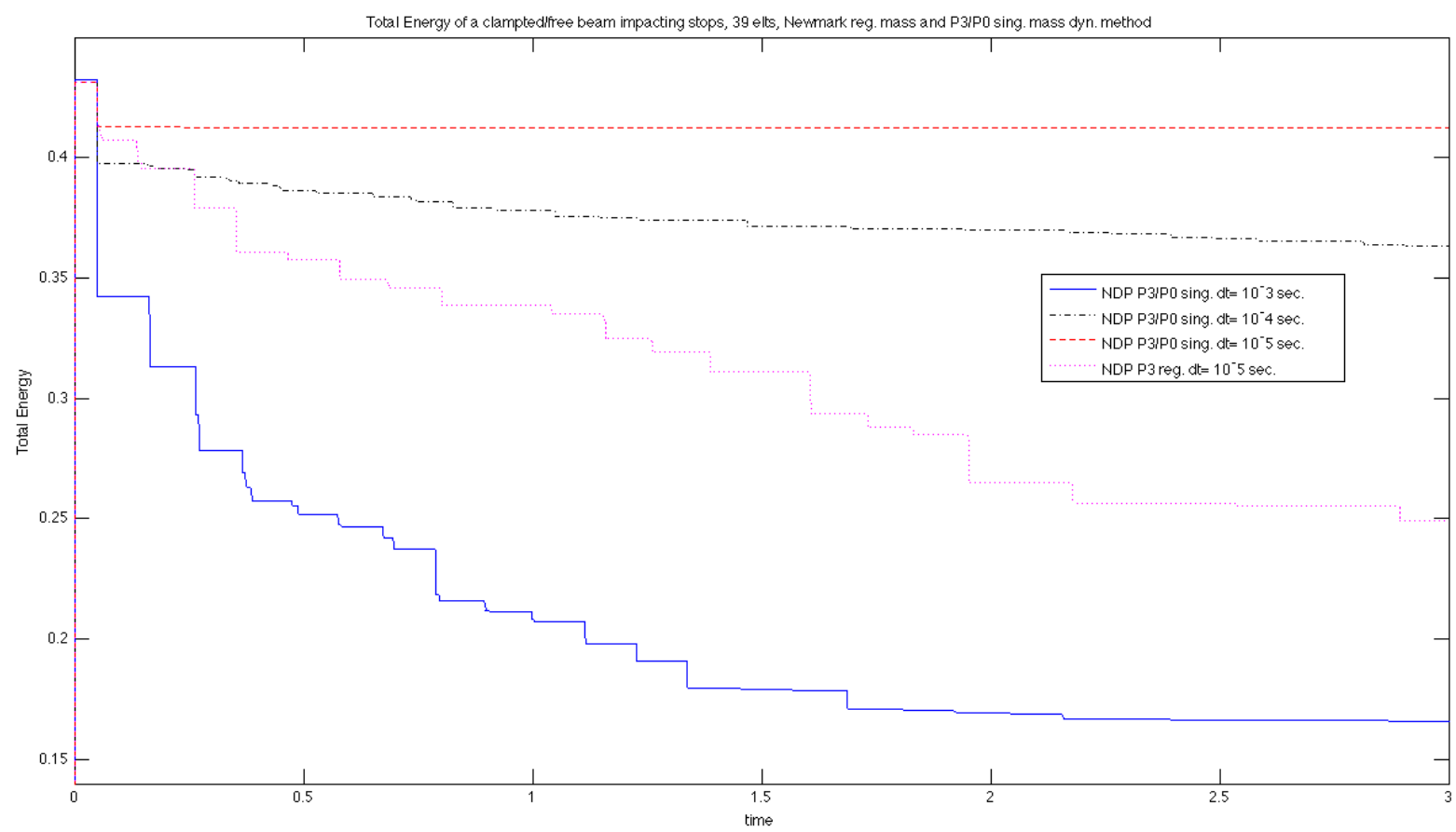

FIGURE 15. Energy for different time steps - Regular versus P3/P0 singular mass matrix for NDP scheme. $e=0, \Delta t=10^{-3}, 10^{-4}, 10^{-5}, 39$ elements. 


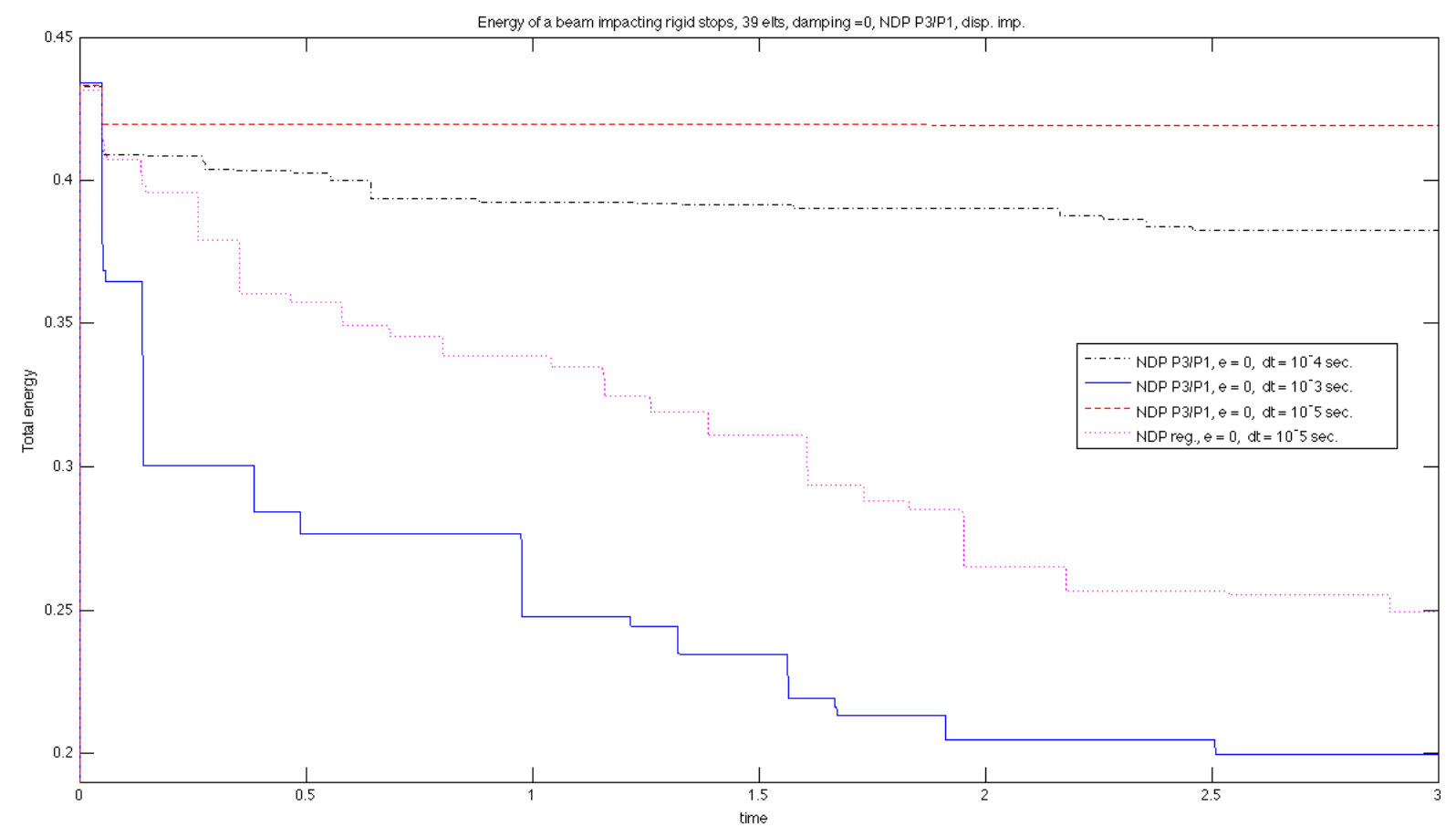

FiguRE 16. Energy for different time steps - Regular versus P3/P1 singular mass matrix for NDP scheme. $e=0, \Delta t=10^{-3}, 10^{-4}, 10^{-5}, 39$ elements.

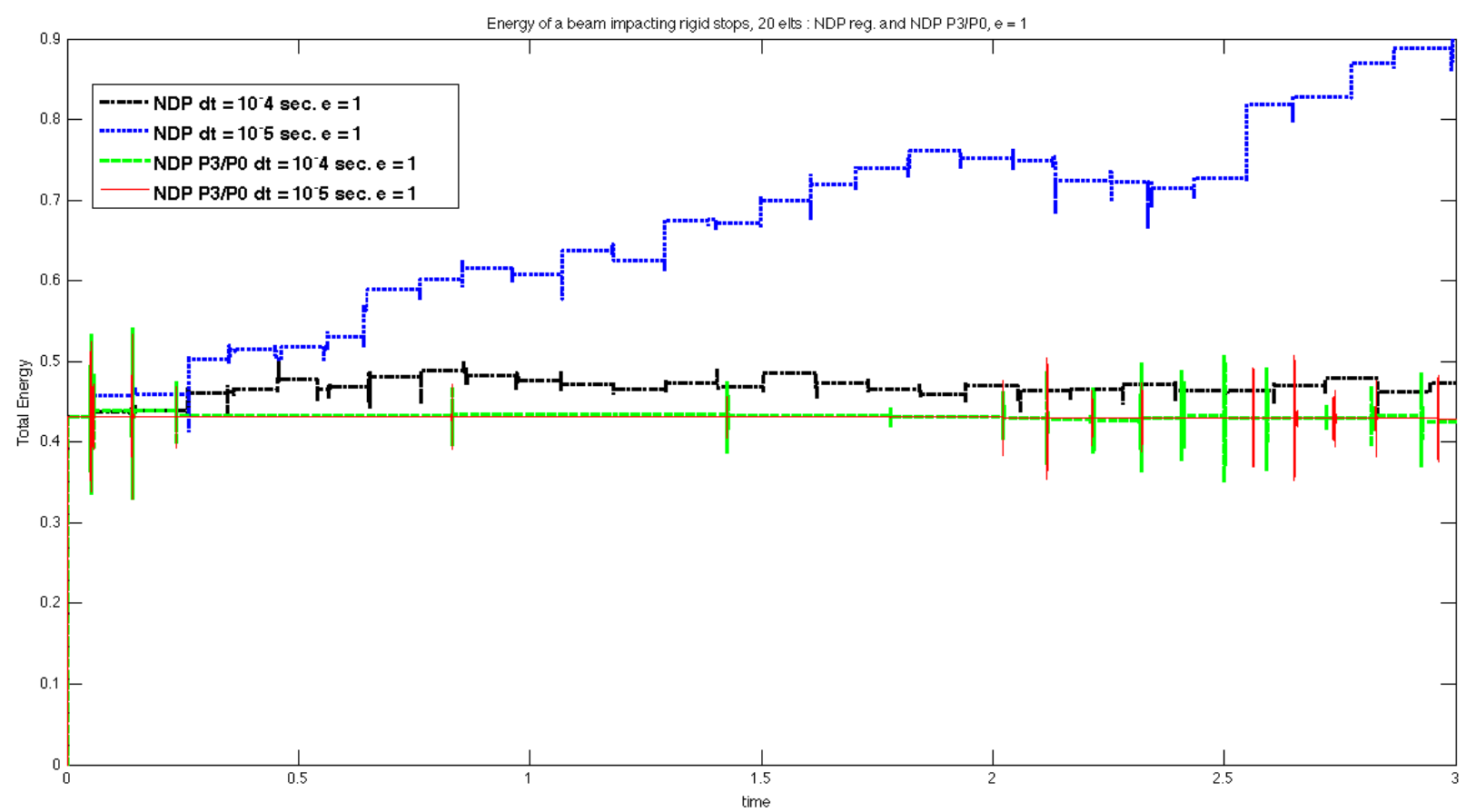

FiguRE 17. Regular versus $\mathrm{P} 3 / \mathrm{P} 0$ singular mass matrix. NDP scheme. $e=1, \Delta t=$ $10^{-4}, 10^{-5}$, 19 elements. 

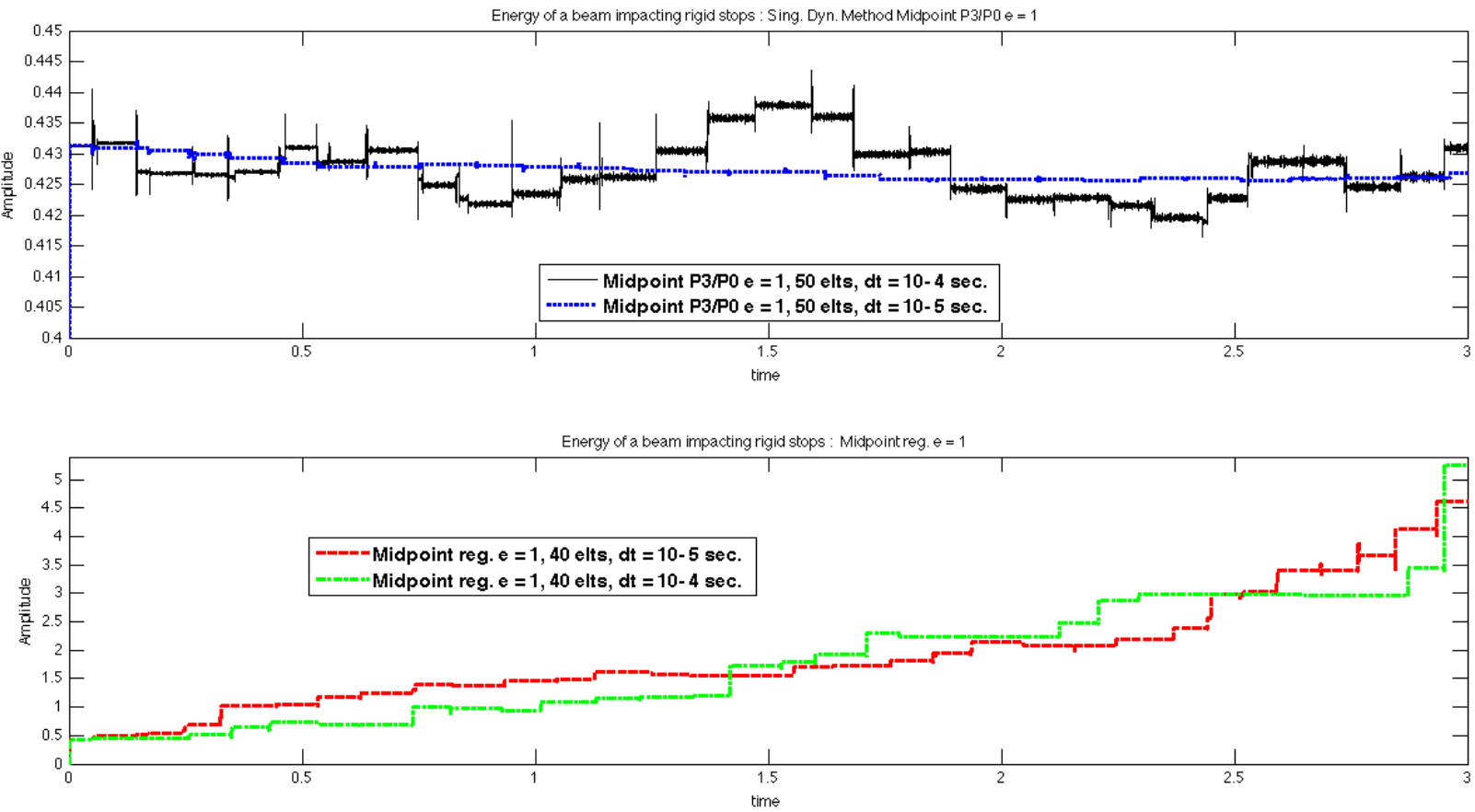

FiguRE 18. Regular versus $\mathrm{P} 3 / \mathrm{P} 0$ singular mass matrix. Midpoint scheme. $e=1, \Delta t=$ $10^{-4}, 10^{-5}, 40$ and 50 elements.

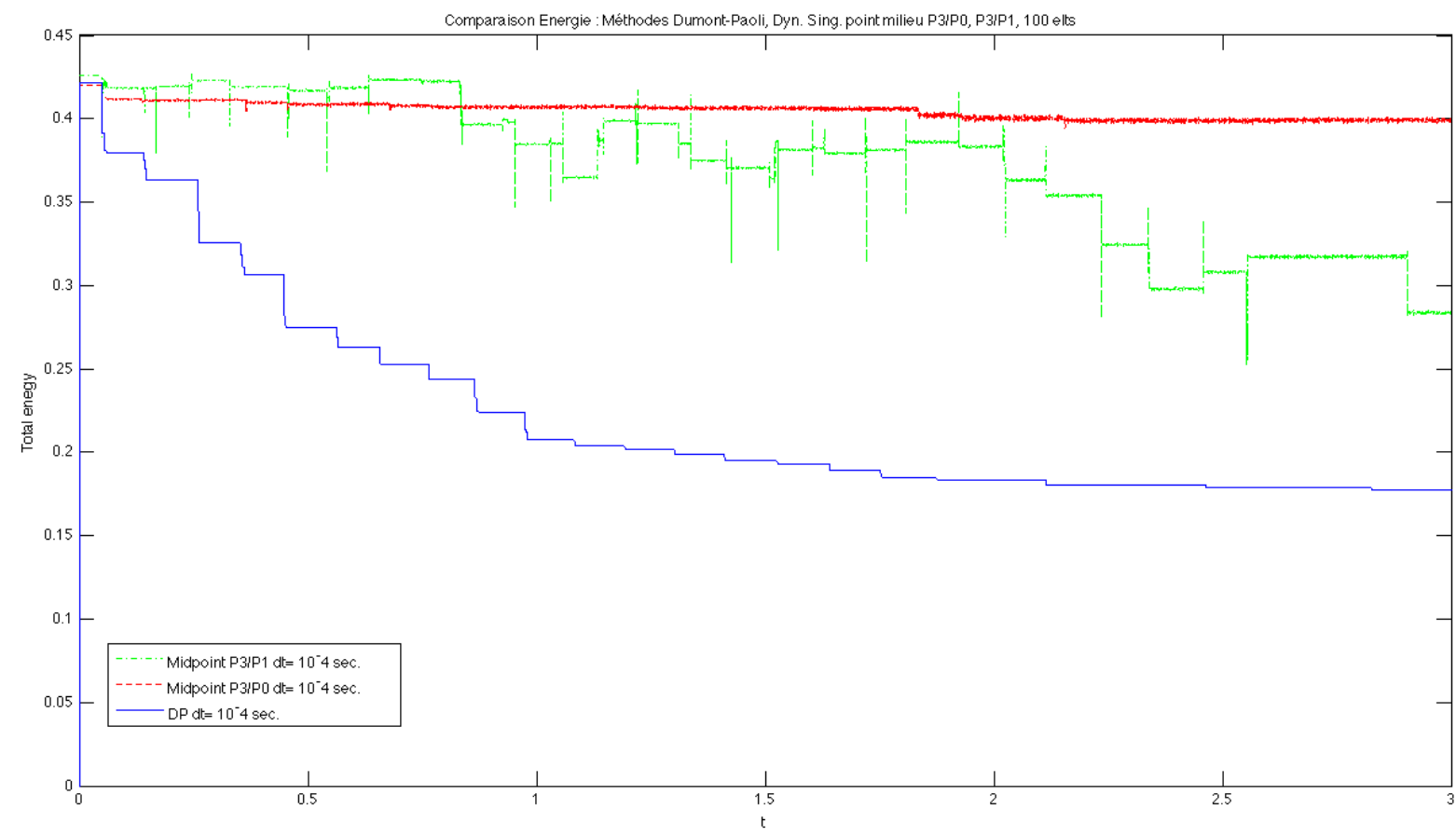

Figure 19. Regular NDP scheme $(e=0)$ versus $\mathrm{P} 3 / \mathrm{P} 0$ and $\mathrm{P} 3 / \mathrm{P} 1$ midpoint schemes $(e=1)$. $\Delta t=10^{-4}, 100$ elements. 


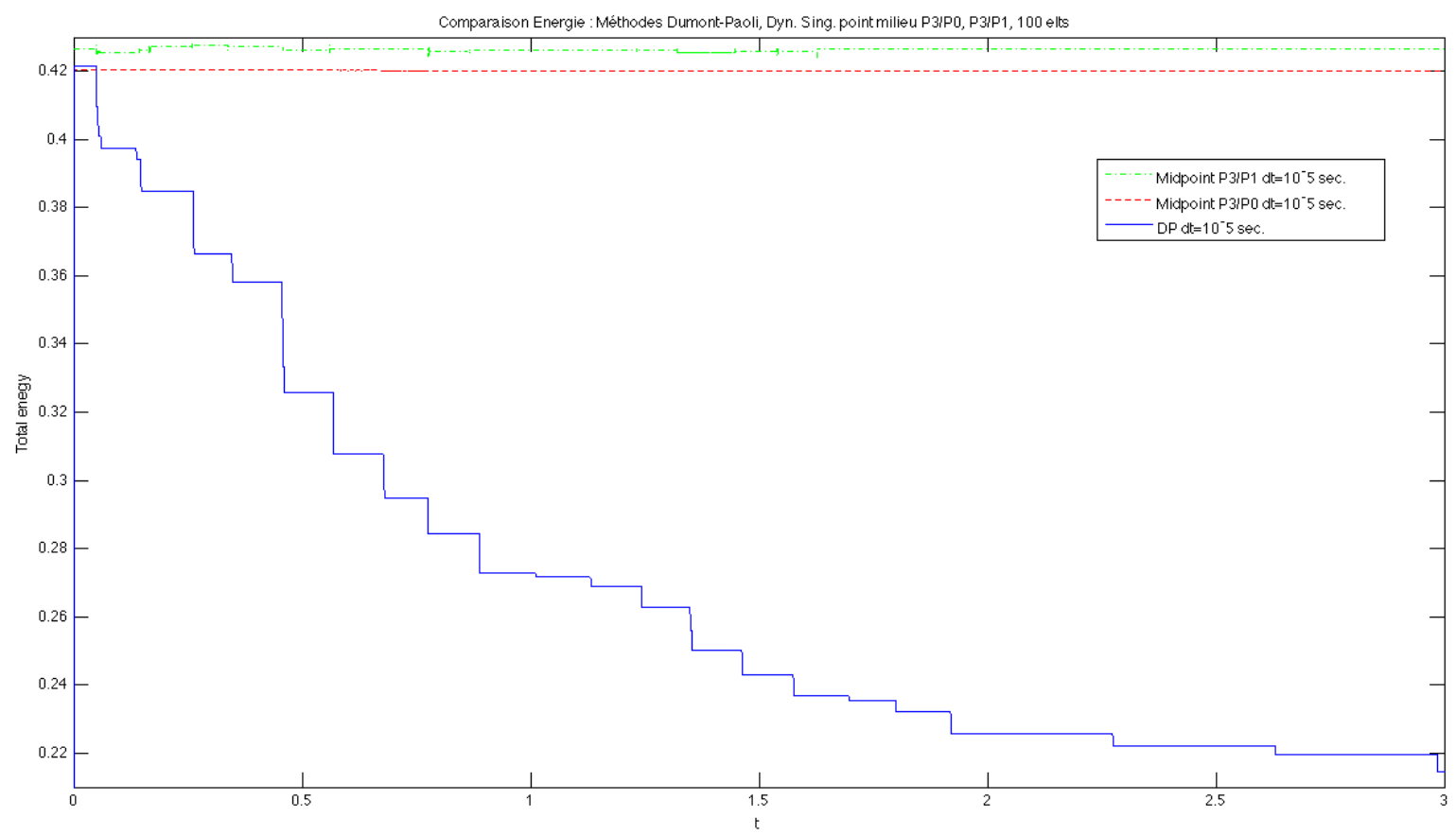

Figure 20. Regular NDP scheme $(e=0)$ versus $\mathrm{P} 3 / \mathrm{P} 0$ and $\mathrm{P} 3 / \mathrm{P} 1$ midpoint schemes $(e=1)$. $\Delta t=10^{-5}, 100$ elements.

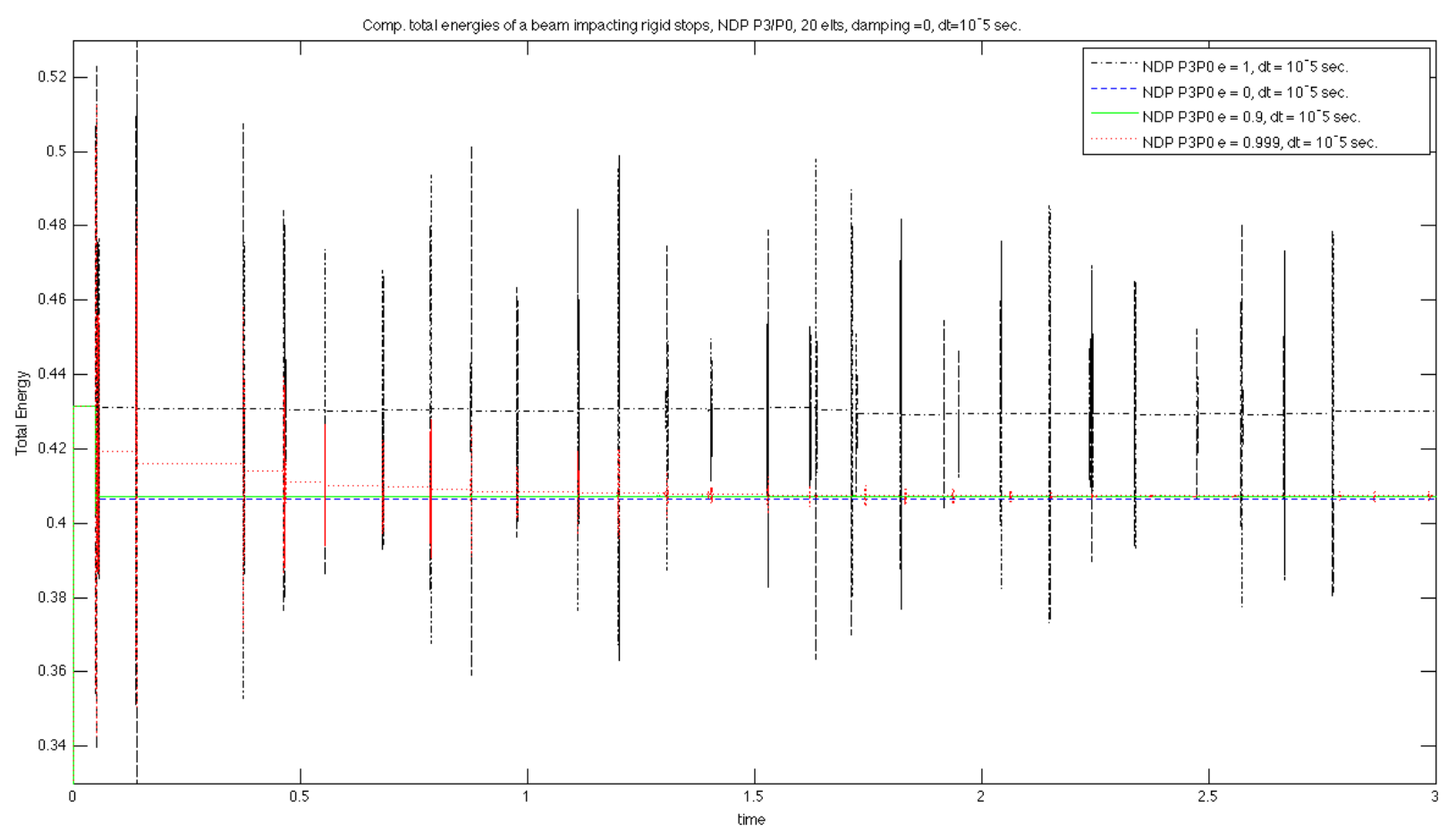

Figure 21. Effect of values of $e . e=0,0.9,0.999,1 . \mathrm{P} 3 / \mathrm{P} 0$ singular mass matrix NDP scheme. $\Delta t=10^{-5}, 20$ elements. 


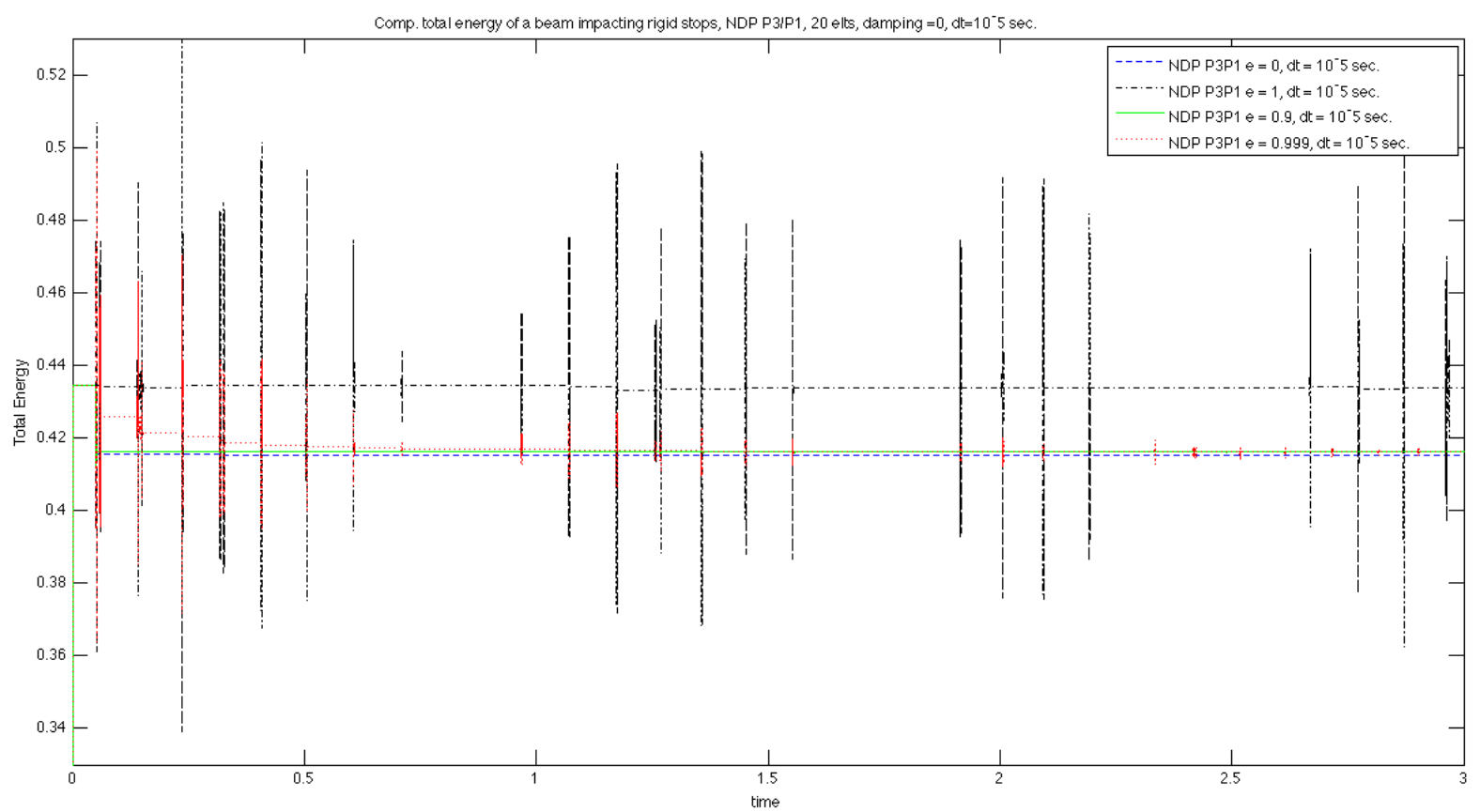

Figure 22. Effect of values of $e . e=0,0.9,0.999,1$. P3/P1 singular mass matrix NDP scheme. $\Delta t=10^{-5}, 20$ elements.

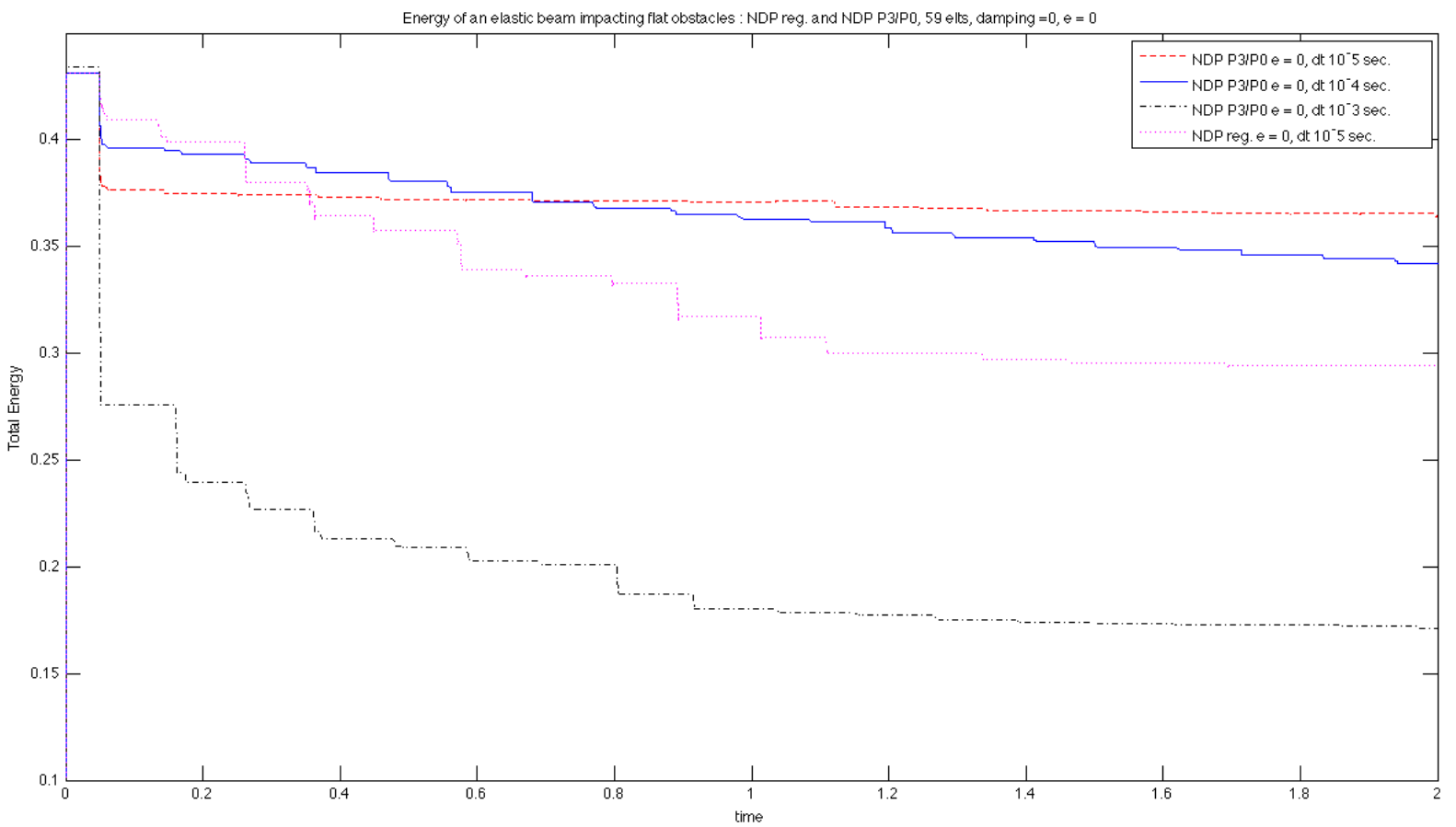

Figure 23. Energy for different time steps - Regular versus P3/P0 singular mass matrix for NDP scheme. $e=0, \Delta t=10^{-3}, 10^{-4}, 10^{-5}, 59$ elements. 


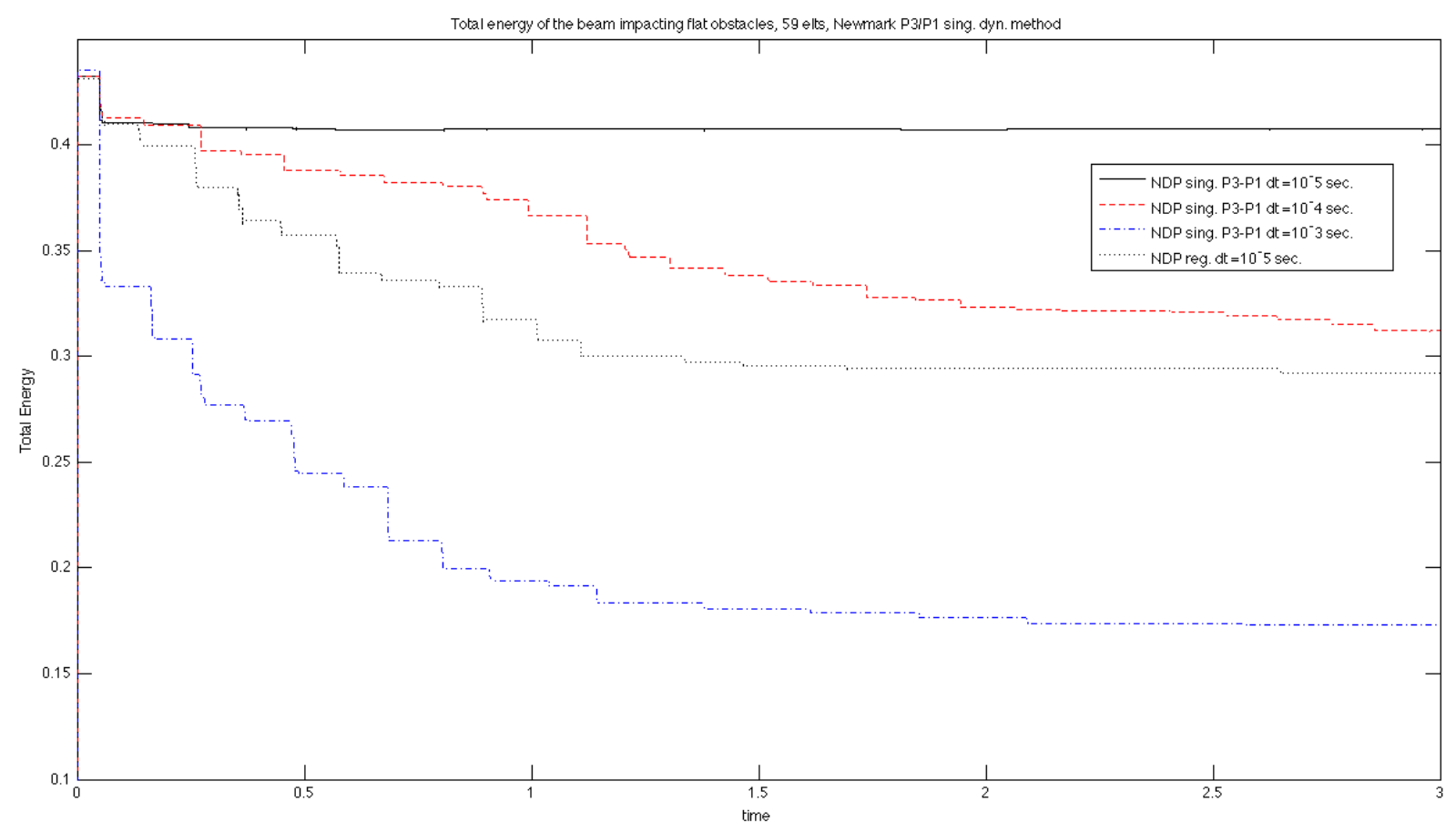

Figure 24. Energy for different time steps - Regular versus P3/P1 singular mass matrix for NDP scheme. $e=0, \Delta t=10^{-3}, 10^{-4}, 10^{-5}, 59$ elements.

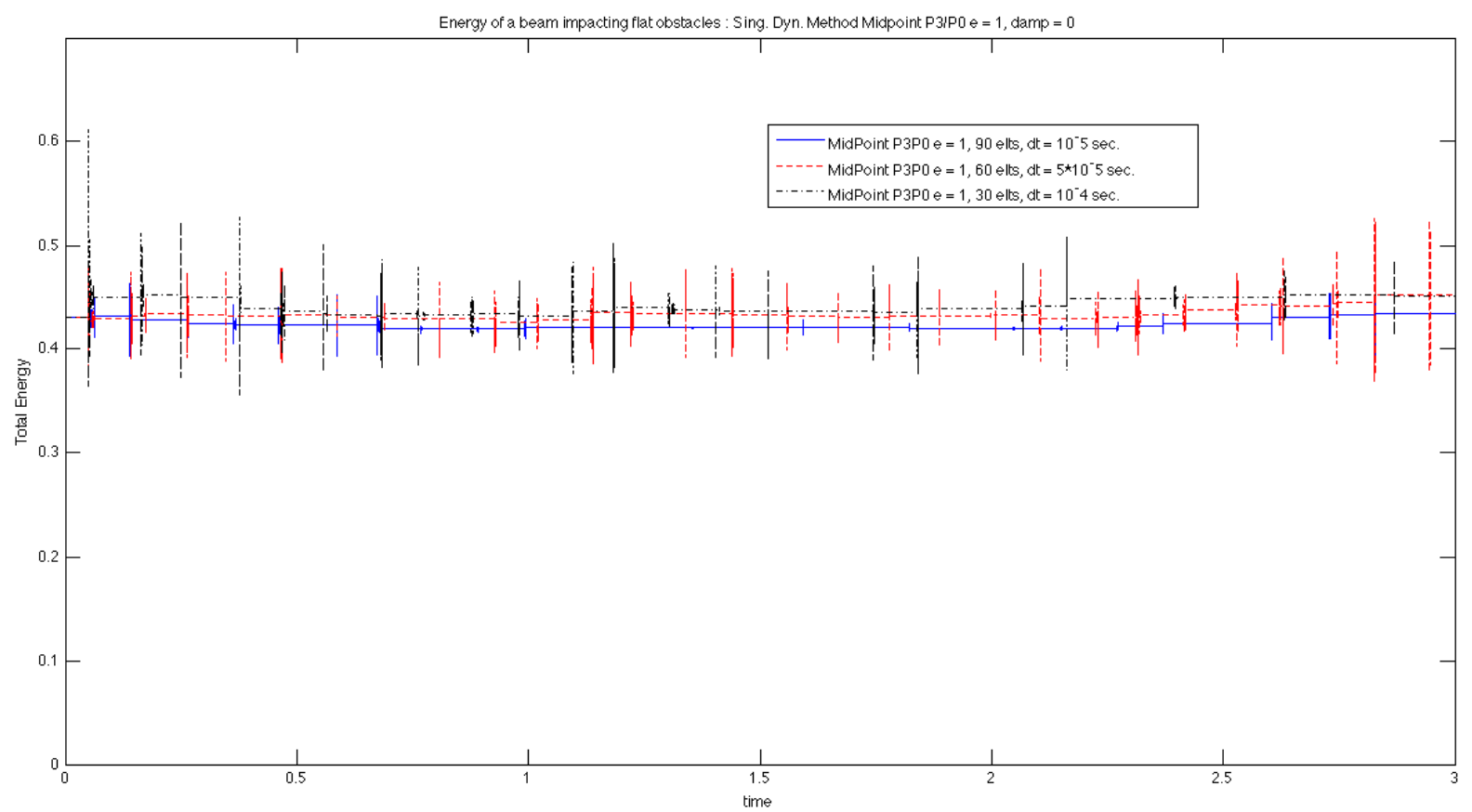

Figure 25. Energy for different time steps - P3/P0 singular mass matrix for midpoint scheme. $e=1, \Delta t=10^{-4}, 5.10^{-5}, 10^{-5}, 30 / 60 / 90$ elements. 


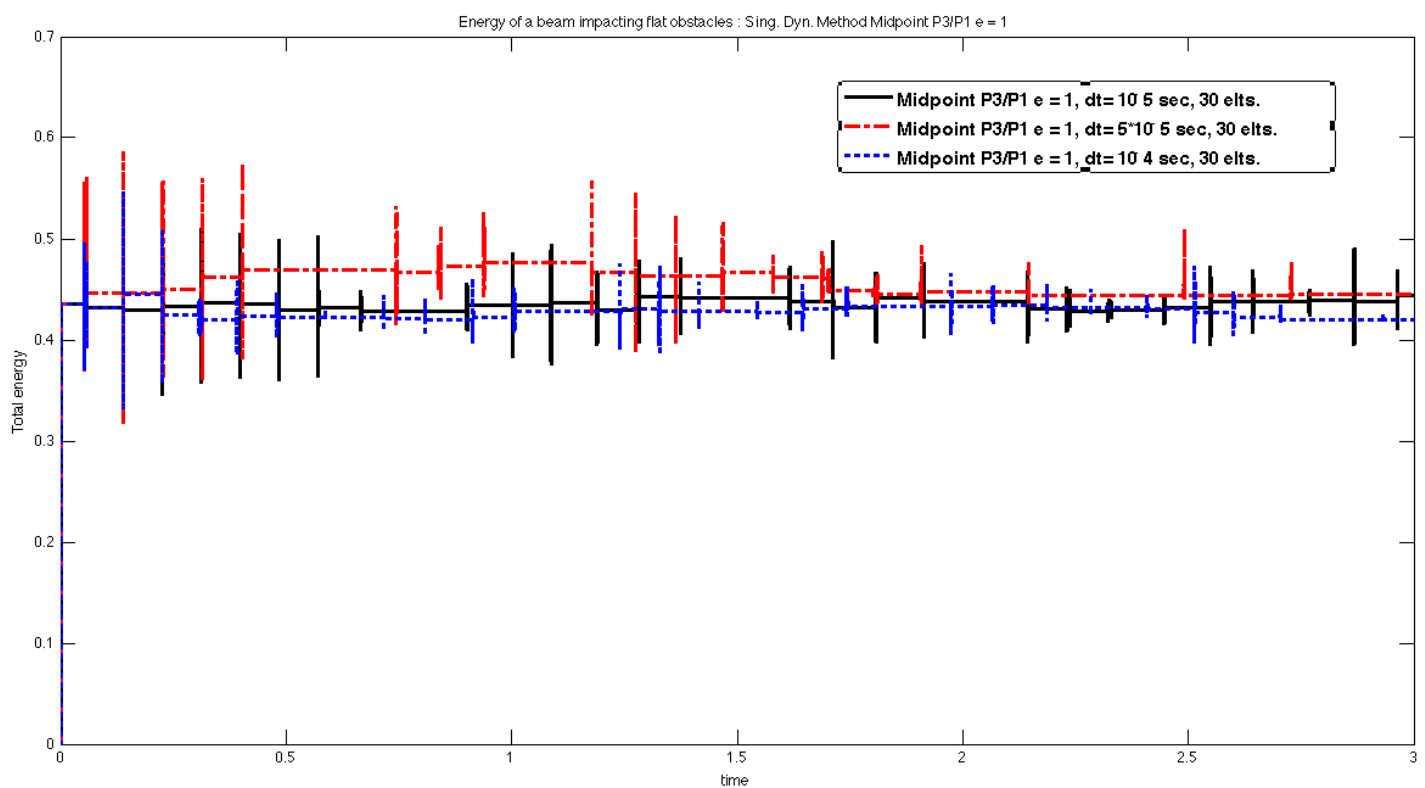

FiguRE 26. Energy for different time steps - P3/P1 singular mass matrix for midpoint scheme. $e=1, \Delta t=10^{-4}, 5.10^{-5}, 10^{-5}, 30$ elements.

Then, for singular mass matrix Newmark-Dumont-Paoli schemes, the effect of values of $e$ is studied. The figures exhibit that, except when $e=1$, a small loss of energy occurs at the beginning of the motion, even for values of $e$ close to 1 .

\subsubsection{Case of flat obstacles}

The case of a beam between two symmetric flat obstacles is considered again. The figures allows to see that the effect of the choice of the time step and/or the restitution coefficient is very similar to what was obtained in the case of pointwise obstacles.

\section{Conclusion}

This paper presents an application of the singular dynamic method to fourth order beam impact problems. This strategy has proved its capabilities as illustrated by numerous numerical results. The following of this work is to improve this model by taking into account friction and damping. Furthermore, we are currently working on extension of this study to dynamical impact of thin plates, using the Kirchhoff-Love model. Finally, using restitution coefficient for updating numerical models will also be investigated in a forthcoming paper.

\section{REFERENCES}

[1] J. Ahn and D.E. Stewart, An Euler-Bernoulli beam with dynamic contact: discretization, convergence and numerical results. SIAM J. Numer. Anal. 43 (2005) 1455-1480.

[2] N.J. Carpenter, Lagrange constraints for transient finite element surface contact. Internat. J. Numer. Methods Engrg. 32 (1991) 103-128.

[3] P. Deuflhard, R. Krause and S. Ertel, A contact-stabilized Newmark method for dynamical contact problems. Internat. J. Numer. Methods Engrg. 73 (2007) 1274-1290.

[4] Y. Dumont and L. Paoli, Vibrations of a beam between obstacles: convergence of a fully discretized approximation. ESAIM: M2AN 40 (2006) 705-734.

[5] Y. Dumont and L. Paoli, Numerical simulation of a model of vibrations with joint clearance. Int. J. Comput. Appl. Technol. 33 (2008) 41-53. 
[6] P. Hauret and P. Le Tallec, Energy controlling time integration methods for nonlinear elastodynamics and low-velocity impact. Comput. Methods Appl. Mech. Eng. 195 (2006) 4890-4916.

[7] H.B. Khenous, P. Laborde and Y. Renard, Mass redistribution method for finite element contact problems in elastodynamics. Eur. J. Mech. A. Solids 27 (2008) 918-932.

[8] K. Kuttler and M. Shillor, Vibrations of a beam between two stops, Dynamics of Continuous, Discrete and Impulsive Systems, Series B. Applications and Algorithms 8 (2001) 93-110.

[9] T.A. Laursen and V. Chawla, Design of energy conserving algorithms for frictionless dynamic contact problems. Internat. J. Numer. Methods Engrg. 40 (1997) 863-886.

[10] T.A. Laursen and G.R. Love, Improved implicit integrators for transient impact problems-geometric admissibility within the conserving framework. Internat. J. Numer. Methods Engrg. 53 (2002) 245-274.

[11] L. Paoli, Time discretization of vibro-impact. Philos. Trans. Roy. Soc. London A 359 (2001) 2405-2428.

[12] L. Paoli and M. Schatzman, A numerical scheme for impact problems. I. The one-dimensional case. SIAM J. Numer. Anal. 40 (2002) 702-733.

[13] L. Paoli and M. Schatzman, Numerical simulation of the dynamics of an impacting bar. Comput. Methods Appl. Mech. Eng. 196 (2007) 2839-2851.

[14] A. Petrov and M. Schatzman, Viscolastodynamique monodimensionnelle avec conditions de Signorini. C. R. Acad. Sci. Paris, I 334 (2002) 983-988.

[15] A. Petrov and M. Schatzman, A pseudodifferential linear complementarity problem related to a one dimensional viscoelastic model with Signorini condition. Arch. Rational Mech. Anal., to appear.

[16] Y. Renard, The singular dynamic method for constrained second order hyperbolic equations. Application to dynamic contact problems. J. Comput. Appl. Math. 234 (2010) 906-923.

[17] R.L. Taylor and P. Papadopoulos, On a finite element method for dynamic contact-impact problems. Internat. J. Numer. Methods Engrg. 36 (1993) 2123-2140. 